Pure and Applied Mathematics Quarterly

Volume 8, Number 4

$1147-1177,2012$

\title{
Hodge Integral Identities from the Cut-and-Join Equation of Mariño-Vafa Formula
}

\author{
Shengmao Zhu
}

\begin{abstract}
In this paper, we calculate the Laplace transform of cut-andjoin equation of Mariño-Vafa formula and obtain a polynomial identity of Hodge integral. Subsequently, we present how to obtain some Hodge integral identities from this polynomial identity. Lastly, we will give a recursion formula for Hodge integral $\left\langle\tau_{b_{L}} \lambda_{g} \lambda_{1}\right\rangle_{g}$ where $b_{L}=\left(b_{1}, \ldots, b_{l}\right)$.
\end{abstract}

Keywords: Hodge integral, Cut-and-join equation, Mariño-Vafa formula, Recursion formula.

\section{Contents}

1. Introduction

2. The Laplace transform of cut-and-join equation of Mariño-Vafa formula

2.1. The Laplace transform of the generating function $\mathcal{C}_{g, \mu}(\tau)$

2.2. The Laplace transform of the cut-and-join equation

3. Some Hodge integral identities

3.1. Preliminary calculations

3.2. Expansion of $\tau$ at $\infty$

3.3. Expansion of $\tau$ at 0

3.4. A recursion formula for Hodge integral $\left\langle\tau_{b_{L}} \lambda_{g} \lambda_{1}\right\rangle_{g}$ 
4. Conclusion

\section{INTRODUCTION}

In a recent paper [7], B. Eynard, M. Mulase and B. Safnuk stated the Laplace transform of the cut-and-join equation of the partition function of Hurwitz numbers and obtained a polynomial identity of linear Hodge integrals. As an application, they proved the Bouchard-Mariño conjecture on Hurwitz numbers [2]. Then, M. Mulase and N. Zhang [14] showed that this polynomial identity can also be used to derive the DVV equation and $\lambda_{g}$-integral with the same method in $[9]$.

In 2003, C.-C. Liu, K. Liu and J. Zhou [11] proved the celebrated Mariño-Vafa conjecture [13]. One of the main step in their proof is to show the generating function of Hodge integral with triple $\lambda$-classes $\mathcal{C}(\lambda, p ; \tau)$ satisfies the cut-andjoin equation. Combining the cut-and-join equation of the combinatorial side [16], they finished the proof of Mariño-Vafa formula. Moreover, the famous ELSV formula [6] for Hurwitz numbers is the large framing limit of Mariño-Vafa formula $[12]$.

With the above motivations, we calculate the Laplace transform of the cutand-join equation for Mariño-Vafa formula in the first part of this paper. The main result is

Theorem 1.1. For $g \geq 1$ and $l \geq 1$, we have the following equation:

$$
\begin{aligned}
(1) \quad & -\left(\tau^{2}+\tau\right)^{l-2} \sum_{b_{L} \geq 0}\left((l-1)(2 \tau+1)\left\langle\tau_{b_{L}} \Gamma_{g}(\tau)\right\rangle_{g}+\left(\tau^{2}+\tau\right)\left\langle\tau_{b_{L}} \frac{d}{d \tau} \Gamma_{g}(\tau)\right\rangle_{g}\right) \\
& \cdot \hat{\Psi}_{b_{L}}\left(t_{L} ; \tau\right)-\left(\tau^{2}+\tau\right)^{l-1} \sum_{b_{L} \geq 0}\left\langle\tau_{b_{L}} \Gamma_{g}(\tau)\right\rangle_{g} \\
& \cdot \sum_{i=1}^{l}\left(\frac{\partial}{\partial \tau} \hat{\Psi}_{b_{i}}\left(t_{i} ; \tau\right)+\frac{1}{t_{i} \tau+1} \hat{\Psi}_{b_{i}+1}\left(t_{i} ; \tau\right)\right) \hat{\Psi}_{b_{L \backslash\{i\}}}\left(t_{L \backslash\{i\}} ; \tau\right) \\
& =-\frac{\left(\tau^{2}+\tau\right)^{l-2}}{\tau+1} \sum_{1 \leq i<j \leq l} \sum_{\substack{a \geq 0 \\
b_{L} \backslash\{i, j\}}}\left\langle\tau_{a} \tau_{b_{L} \backslash\{i, j\}} \Gamma_{g}(\tau)\right\rangle_{g} \hat{\Psi}_{b_{L \backslash\{i, j\}}}\left(t_{L \backslash\{i, j\}} ; \tau\right)
\end{aligned}
$$




$$
\begin{aligned}
& \frac{\left(t_{j}-1\right)\left(t_{i}^{2} \tau+t_{i}\right) \hat{\Psi}_{a+1}\left(t_{i} ; \tau\right)-\left(t_{i}-1\right)\left(t_{j}^{2} \tau+t_{j}\right) \hat{\Psi}_{a+1}\left(t_{j} ; \tau\right)}{t_{i}-t_{j}} \\
& +\frac{\left(\tau^{2}+\tau\right)^{l-1}}{2} \sum_{i=1}^{l} \sum_{\substack{a_{1} \geq 0 \\
a_{2} \geq 0 \\
b_{L} \backslash\{i\} \geq 0}}\left(\left(\tau^{2}+\tau\right)\left\langle\tau_{a_{1}} \tau_{a_{2}} \tau_{b_{L} \backslash\{i\}} \Gamma_{g-1}(\tau)\right\rangle_{g-1}\right. \\
& \left.-\sum_{\substack{g_{1}+g_{2}=g \\
\mathcal{I} \amalg \mathcal{J}=L \backslash\{i\} \\
2 g_{1}-1+|\mathcal{I}|>0 \\
2 g_{2}-1+|\mathcal{J}|>0}}\left\langle\tau_{a_{1}} \tau_{b_{\mathcal{I}}} \Gamma_{g_{1}}(\tau)\right\rangle_{g_{1}}\left\langle\tau_{a_{2}} \tau_{b_{\mathcal{J}}} \Gamma_{g_{2}}(\tau)\right\rangle_{g_{2}}\right) \\
& \cdot \prod_{n=1}^{2} \hat{\Psi}_{a_{n}+1}\left(t_{i} ; \tau\right) \hat{\Psi}_{b_{L \backslash\{i\}}}\left(t_{L \backslash\{i\}} ; \tau\right)
\end{aligned}
$$

where $L=\{1,2, . ., l\}$ is an index set, and for any subset $I \subset L$, we denote

$$
t_{I}=\left(t_{i}\right)_{i \in I}, \quad b_{I}=\left(b_{i}\right)_{i \in I}, \tau_{b_{I}}=\prod_{i \in I} \tau_{b_{i}}, \hat{\Psi}_{b_{I}}\left(t_{I}, \tau\right)=\prod_{i \in I} \hat{\Psi}_{b_{i}}\left(t_{i}, \tau\right)
$$

and $\Gamma_{g}(\tau)=\Lambda_{g}^{\vee}(1) \Lambda_{g}^{\vee}(-\tau-1) \Lambda_{g}^{\vee}(\tau), \hat{\Psi}_{n}(t ; \tau)=\left(\frac{\left(t^{2}-t\right)(t \tau+1)}{\tau+1} \frac{d}{d t}\right)^{n}\left(\frac{t-1}{\tau+1}\right)$ for $n \geq$ 0 .

We remark that Theorem 1.1 is equivalent to the symmetrized cut-and-join equation of Mariño-Vafa formula obtained by L. Chen [3].

Furthermore, we use the result of Theorem 1.1 to obtain some Hodge integral identities. In fact, $\hat{\Psi}_{b}(t, \tau)$ can be written as

$$
\hat{\Psi}_{b}(t ; \tau)=\sum_{k=0}^{b} \frac{\tau^{k}}{(\tau+1)^{b+1}} \Psi_{b}^{k}(t)
$$

and $\Psi_{b}^{k}(t), 0 \leq k \leq b$ could be calculated by definition.

Firstly, we consider the expansion of $\tau$ at $\infty$. Taking the highest level of formula (1), we get

\section{Corollary 1.2.}

$$
\sum_{b_{L} \geq 0}\left\langle\tau_{b_{L}} \Lambda_{g}^{\vee}(1)\right\rangle\left((2 g-2+l) \Psi_{b_{L}}^{b_{L}}\left(t_{L}\right)+\sum_{i=1}^{l}\left(t_{i}^{2}-t_{i}\right) \frac{\partial}{\partial t_{i}} \Psi_{b_{i}}^{b_{i}}\left(t_{i}\right) \Psi_{b_{L \backslash\{i\}}}^{b_{L \backslash i\}}}\left(t_{L \backslash\{i\}}\right)\right)
$$




$$
\begin{aligned}
& =\sum_{1 \leq i<j \leq l} \sum_{\substack{a \geq 0 \\
b_{L \backslash\{i, j\}} \geq 0}}\left\langle\tau_{a} \tau_{b_{L \backslash\{i, j\}}} \Lambda_{g}^{\vee}(1)\right\rangle_{g} \Psi_{b_{L \backslash\{i, j\}}}^{b_{L \backslash i, j\}}}\left(t_{L \backslash\{i, j\}}\right) \\
& \frac{\left(t_{j}-1\right) t_{i}^{2} \Psi_{a+1}^{a+1}\left(t_{i}\right)-\left(t_{i}-1\right) t_{j}^{2} \Psi_{a+1}^{a+1}\left(t_{j}\right)}{t_{i}-t_{j}} \\
& +\frac{1}{2} \sum_{i=1}^{l} \sum_{\substack{a_{1} \geq 0 \\
a_{2} \geq 0 \\
b_{L} \backslash\{i\} \geq 0}}\left(\left\langle\tau_{a_{1}} \tau_{a_{2}} \tau_{b_{L \backslash\{i\}}} \Lambda_{g-1}^{\vee}(1)\right\rangle_{g-1}+\sum_{\substack{g_{1}+g_{2}=g \\
\mathcal{I} \cup \mathcal{J}=L \backslash\{i\} \\
2 g_{1}-1+|\mathcal{I}|>0 \\
2 g_{2}-1+|\mathcal{J}|>0}}\left\langle\tau_{a_{1}} \tau_{b_{\mathcal{I}}} \Lambda_{g_{1}}^{\vee}(1)\right\rangle_{g_{1}}\right. \\
& \left.\cdot\left\langle\tau_{a_{2}} \tau_{b_{\mathcal{J}}} \Lambda_{g_{2}}^{\vee}(1)\right\rangle_{g_{2}}\right) \Psi_{a_{1}+1}^{a_{1}+1}\left(t_{i}\right) \Psi_{a_{2}+1}^{a_{2}+1}\left(t_{i}\right) \Psi_{b_{L \backslash\{i\}}}^{b_{L \backslash\{i\}}}\left(t_{L \backslash\{i\}}\right) .
\end{aligned}
$$

We show in Section 2 that $\Psi_{b}^{b}(t)$ is equal to $\hat{\xi}_{b}(t)$ which is defined by $\hat{\xi}_{b}(t)=$ $\left(\left(t^{3}-t^{2}\right) \frac{d}{d t}\right)^{b}(t-1)$ in formula (1.2) in [7]. Hence, the main Theorem 1.1 in [7] is the special case of formula (1) in this paper. Similarly, taking the sub-highest level of Theorem 1.1, we also get another Hodge integral identity in Section 3.

Next, we consider the expansion of $\tau$ at $\tau=0$. In this case, the lowest level of formula (1) is

\section{Corollary 1.3.}

$$
\begin{aligned}
& \sum_{b_{L} \geq 0}\left\langle\tau_{b_{L}} \lambda_{g}\right\rangle_{g} \Psi_{b_{L}}^{0}\left(t_{L}\right)=\frac{1}{l-1} \sum_{1 \leq i<j \leq l} \sum_{\substack{a \geq 0 \\
b_{L \backslash\{i, j\}} \geq 0}}\left\langle\tau_{a} \tau_{b_{L} \backslash\{i, j\}} \lambda_{g}\right\rangle_{g} \Psi_{b_{L \backslash\{i, j\}}}^{0}\left(t_{L \backslash\{i, j\}}\right) \\
& \cdot \frac{\left(t_{j}-1\right) t_{i}^{2} \Psi_{a+1}^{0}\left(t_{i}\right)-\left(t_{i}-1\right) t_{j}^{2} \Psi_{a+1}^{0}\left(t_{j}\right)}{t_{i}-t_{j}} .
\end{aligned}
$$

We can rederive the $\lambda_{g}$-integral [8] from Corollary 1.3.

Furthermore, we also pick up the sub-lowest level of formula (1) and get the following identity:

\section{Corollary 1.4.}

$$
\sum_{b_{L} \geq 0}\left\langle\tau_{b_{L}} \lambda_{g}\right\rangle_{g} l\left(-\left(\left|b_{L}\right|+1\right) \Psi_{b_{L}}^{0}\left(t_{L}\right)+\sum_{j=1} \Psi_{b_{j}}^{1}\left(t_{j}\right) \Psi_{b_{L \backslash\{j\}}}^{0}\left(t_{L \backslash\{j\}}\right)\right)
$$




$$
\begin{aligned}
& +\sum_{b_{L} \geq 0}\left\langle\tau_{b_{L}} \lambda_{g}\right\rangle_{g} \sum_{i=1}^{l}\left(t_{i}^{2}-t_{i}\right) \frac{\partial}{\partial t_{i}} \Psi_{b_{i}}^{0}\left(t_{i}\right) \Psi_{b_{L \backslash\{i\}}}^{0}\left(t_{L \backslash\{i\}}\right) \\
& +\sum_{b_{L} \geq 0}\left\langle\tau_{b_{L}} \sum_{d=g-1}^{3 g-3} P_{d}(\lambda)\right\rangle_{g} l \Psi_{b_{L}}^{0}\left(t_{L}\right) \\
& =\sum_{1 \leq i<j \leq l} \sum_{\substack{a \geq 0 \\
b_{L} \backslash\{i, j\} \geq 0}}\left\langle\tau_{a} \tau_{b_{L \backslash\{i, j\}}} \lambda_{g}\right\rangle_{g} \\
& \cdot\left(\frac{\left(t_{j}-1\right) t_{i}\left(\Psi_{a+1}^{1}\left(t_{i}\right)+\left(t_{i}-\left|b_{L \backslash\{i, j\}}\right|-a-3\right) \Psi_{a+1}^{0}\left(t_{i}\right)\right)}{t_{i}-t_{j}}\right. \\
& \left.-\frac{\left(t_{i}-1\right) t_{j}\left(\Psi_{a+1}^{1}\left(t_{j}\right)+\left(t_{j}-\left|b_{L \backslash\{i, j\}}\right|-a-3\right) \Psi_{a+1}^{0}\left(t_{j}\right)\right)}{t_{i}-t_{j}}\right) \Psi_{b_{L \backslash\{i, j\}}}^{0}\left(t_{L \backslash\{i, j\}}\right) \\
& +\sum_{1 \leq i<j \leq l} \sum_{\substack{a \geq 0 \\
b_{L} \backslash\{i, j\} \geq 0}}\left\langle\tau_{a} \tau_{b_{L \backslash\{i, j\}}} \lambda_{g}\right\rangle_{g} \sum_{r \neq i, j} \Psi_{b_{r}}^{1}\left(t_{r}\right) \Psi_{b_{L \backslash\{i, j\}}}^{0}\left(t_{L \backslash\{i, j\}}\right) \\
& \cdot \frac{\left(t_{j}-1\right) t_{i} \Psi_{a+1}^{0}\left(t_{i}\right)-\left(t_{i}-1\right) t_{j} \Psi_{a+1}^{0}\left(t_{j}\right)}{t_{i}-t_{j}}+\sum_{1 \leq i<j \leq l} \sum_{\substack{a \geq 0 \\
b_{L \backslash\{i, j\}} \geq 0}}\left\langle\tau_{a} \tau_{b_{L \backslash\{i, j\}}} \sum_{d=g-1}^{3 g-3} P_{d}(\lambda)\right\rangle_{g} \\
& \cdot \Psi_{b_{L \backslash\{i, j\}}^{0}}^{0}\left(t_{L \backslash\{i, j\}}\right) \frac{\left(t_{j}-1\right) t_{i} \Psi_{a+1}^{0}\left(t_{i}\right)-\left(t_{i}-1\right) t_{j} \Psi_{a+1}^{0}\left(t_{j}\right)}{t_{i}-t_{j}} \\
& +\frac{1}{2} \sum_{i=1}^{l} \sum_{\substack{a_{1} \geq 0 \\
a_{2} \geq 0 \\
b_{L} \backslash\{i\} \geq 0}} \sum_{\substack{g_{1}+g_{2}=g \\
\mathcal{I} \cup \mathcal{J}=L \backslash\{i\} \\
2 g_{1}-1+|\mathcal{I}|>0 \\
2 g_{2}-1+|\mathcal{J}|>0}}\left\langle\tau_{a_{1}} \tau_{b_{\mathcal{I}}} \lambda_{g_{1}}\right\rangle_{g_{1}}\left\langle\tau_{a_{2}} \tau_{b_{\mathcal{J}}} \lambda_{g_{2}}\right\rangle_{g_{2}} \\
& \text {. } \Psi_{a_{1}+1}^{0}\left(t_{i}\right) \Psi_{a_{2}+1}^{0}\left(t_{i}\right) \Psi_{b_{L \backslash\{i\}}}^{0}\left(t_{L \backslash\{i\}}\right) \\
& \text { where } \sum_{d=g-1}^{3 g-3} P_{d}(\lambda)=\Lambda_{g}^{\vee}(1) a_{1}(\lambda) \text { and } a_{1}(\lambda)=\sum_{m=1}^{g} m \lambda_{g-m} \lambda_{g}-(-1)^{g} \Lambda_{g}^{\vee}(-1) \lambda_{g-1} \text {. }
\end{aligned}
$$

The above identity contains Hodge integral of type $\left\langle\tau_{b_{L}} \sum_{d=g-1}^{3 g-3} P_{d}(\lambda)\right\rangle_{g}$. By some direct calculations,

$$
\begin{aligned}
& P_{g-1}(\lambda)=\lambda_{g-1}, P_{g}(\lambda)=g \lambda_{g}, P_{g+1}(\lambda)=-\lambda_{g} \lambda_{1}, \\
& \cdots, P_{3 g-3}(\lambda)=(-1)^{g+1} \lambda_{g} \lambda_{g-1} \lambda_{g-2} .
\end{aligned}
$$

As an application of Corollary 1.4, we get the following Hodge integral recursion. 
Theorem 1.5. If $\sum_{i=1}^{l} b_{i}=2 g-4+l$, there exists a constant $C\left(g, l, b_{1}, . ., b_{l}\right)$ related to $g, l, b_{1}, . ., b_{l}$, such that

$$
\left\langle\tau_{b_{L}} \lambda_{g} \lambda_{1}\right\rangle_{g}=\frac{1}{l} \sum_{1 \leq i<j \leq l}\left\langle\tau_{b_{i}+b_{j}-1} \tau_{b_{L} \backslash\{i, j\}} \lambda_{g} \lambda_{1}\right\rangle_{g} \frac{\left(b_{i}+b_{j}\right) !}{b_{i} ! b_{j} !}+C\left(g, l, b_{1}, . ., b_{l}\right)
$$

where $C\left(g, l, b_{1}, . ., b_{l}\right)$ is a very verbose combinatoric constant.

The initial value $\left\langle\tau_{2 g-3} \lambda_{g} \lambda_{1}\right\rangle_{g}=\frac{1}{12}\left[g(2 g-3) b_{g}+b_{1} b_{g-1}\right]$ has been obtained by Y. Li [10]. Combining the recursion formula in Theorem 1.5, we can get all the Hodge integral $\left\langle\tau_{b_{L}} \lambda_{g} \lambda_{1}\right\rangle_{g}$. Moreover, the Hodge integrals $\left\langle\tau_{b_{L}} P_{d}(\lambda)\right\rangle_{g}$ appeared in Corollary 1.4 could also be calculated via the same method. But the computation will be more complicated.

Acknowledgements. The author would like to thank Professor Kefeng Liu for his encouragement, and bringing the paper [7] to his attention. The author is grateful to the referees for their valuable comments and suggestions which greatly improved the presentation of the content.

\section{The Laplace transform of CUT-And-Join Equation of Mariño-VAFA}

\section{FORMULA}

2.1. The Laplace transform of the generating function $\mathcal{C}_{g, \mu}(\tau)$. At first, we introduce some notations followed by [11]. Let

$$
\Lambda_{g}^{\vee}(t)=t^{g}-\lambda_{1} t^{g-1}+\cdots+(-1)^{g} \lambda_{g}
$$

be the Chern polynomial of $\mathbb{E}^{\vee}$, the dual of the Hodge bundle. For a partition $\mu$ given by

$$
\mu_{1} \geq \mu_{2} \geq \cdots \geq \mu_{l(\mu)}>0
$$

we will use the standard notation $|\mu|=\sum_{i=1}^{l(\mu)} \mu_{i}$.

Let $\Gamma_{g}(\tau)=\Lambda_{g}^{\vee}(1) \Lambda_{g}^{\vee}(-\tau-1) \Lambda_{g}^{\vee}(\tau)$ and we define,

$\mathcal{C}_{g, \mu}(\tau)=-\frac{\sqrt{-1}^{|\mu|+l(\mu)}}{|A u t(\mu)|}[\tau(\tau+1)]^{l(\mu)-1} \prod_{i=1}^{l(\mu)} \frac{\prod_{a=1}^{\mu_{i}-1}\left(\mu_{i} \tau+a\right)}{\left(\mu_{i}-1\right) !} \int_{\overline{\mathcal{M}}_{g, l(\mu)}} \frac{\Gamma_{g}(\tau)}{\prod_{i=1}^{l(\mu)}\left(1-\mu_{i} \psi_{i}\right)}$ 
where the Deligne-Mumford stack $\overline{\mathcal{M}}_{g, l}$ is the moduli space of stable curves satisfying the stability condition $2 g-2+l>0$. For the unstable cases $(g, l)=(0,1)$ and $(0,2)$, we define

$$
\begin{gathered}
\int_{\overline{\mathcal{M}}_{0,1}} \frac{1}{1-\mu \psi}=\frac{1}{\mu^{2}}, \\
\int_{\overline{\mathcal{M}}_{0,2}} \frac{1}{\left(1-\mu_{1} \psi_{1}\right)\left(1-\mu_{2} \psi_{2}\right)}=\frac{1}{\mu_{1}+\mu_{2}} .
\end{gathered}
$$

It follows that

$$
\begin{gathered}
\mathcal{C}_{0, d}(\tau)=-\sqrt{-1} d+1 \frac{1}{\tau} \frac{\prod_{a=0}^{d-1}(d \tau+a)}{d !} d^{-2} \\
\mathcal{C}_{0,\left(\mu_{1}, \mu_{2}\right)}(\tau)=\frac{\sqrt{-1} \mu_{1}+\mu_{2}}{\left|A u t\left(\mu_{1}, \mu_{2}\right)\right|} \frac{\tau+1}{\tau} \prod_{i=1}^{2} \frac{\prod_{a=0}^{\mu_{i}-1}\left(\mu_{i} \tau+a\right)}{\mu_{i} !} \frac{1}{\mu_{1}+\mu_{2}} .
\end{gathered}
$$

The Mariño-Vafa formula proved in [11] gives a direct combinatorial formula associated to representation of symmetric groups for the generation function of $\mathcal{C}_{g, \mu}(\tau)$. But we don't go further to this formula here.

Through a direct calculation, we get

$$
\begin{aligned}
\mathcal{C}_{g, \mu}(\tau) & =-\frac{\sqrt{-1}^{|\mu|+l(\mu)}}{|A u t(\mu)|}[\tau(\tau+1)]^{l(\mu)-1} \\
& \cdot \sum_{\substack{b_{i} \geq 0 \\
i=1, . ., l(\mu)}}\left\langle\prod_{i=1}^{l(\mu)} \tau_{b_{i}} \Gamma_{g}(\tau)\right\rangle_{g} \prod_{i=1}^{l(\mu)} \frac{1}{\tau} \frac{\prod_{a=0}^{\mu_{i}-1}\left(\mu_{i} \tau+a\right)}{\mu_{i} !} \mu_{i}^{b_{i}} .
\end{aligned}
$$

Let $\mathcal{C}_{g}(\mu ; \tau)=|A u t(\mu)| \mathcal{C}_{g, \mu}(\tau)$, we define the Laplace transform of $\mathcal{C}_{g, \mu}(\tau)$ as

$$
\mathcal{C}_{g, l}\left(w_{1}, . ., w_{l}\right)=\sum_{\mu \in \mathbb{N}^{l}} \frac{1}{\sqrt{-1}^{l+|\mu|}} \mathcal{C}_{g}(\mu ; \tau) e^{-\left(\mu_{1} w_{1}+\cdots+\mu_{l} w_{l}\right)}
$$

where we have let $l=l(\mu)$.

In the following of this subsection, we will show that the Laplace transform of $\mathcal{C}_{g, \mu}(\tau)$ has a nice expression in the new variable $t$. We need the following Lagrange inversion theorem, 
Theorem 2.1. Let $\phi(\lambda)$ be an invertible formal power series in the indeterminate $\lambda$. Then the functional equation $y=x \phi(y)$ has a unique formal power series solution $y=y(x)$. Moreover, if $f$ is a formal power series, then

$$
f(y(x))=f(0)+\sum_{n \geq 1} \frac{x^{n}}{n}\left[\lambda^{n-1}\right] \frac{d f(\lambda)}{d \lambda} \phi(\lambda)^{n}
$$

and

$$
\frac{f(y(x))}{y} \frac{x d y(x)}{d x}=\sum_{n \geq 0} x^{n}\left[\lambda^{n}\right] f(\lambda) \phi(\lambda)^{n}
$$

We consider the framed Lambert curve $x=y(1-y)^{\tau}$. Put $\phi(y)=\frac{1}{(1-y)^{\tau}}$ and $f(y)=y$ in formula (3), we have $y=\sum_{k \geq 1} \frac{\prod_{a=0}^{k-2}(k \tau+a)}{k !} x^{k}$. We now introduce the new variables $t, w$ through $t=\frac{1}{1-(\tau+1) y}$ and $x=e^{-w}$. It is easy to see that the derivative of variables $w, x, y, t$ satisfy the following relations:

$$
-\frac{d}{d w}=x \frac{d}{d x}=\frac{(1-y) y}{1-(\tau+1) y} \frac{d}{d y}=\left(\frac{t(t-1)(t \tau+1)}{\tau+1}\right) \frac{d}{d t} .
$$

One can get that

$$
t=1-\frac{(\tau+1) y}{\tau}+\frac{(\tau+1)^{2}}{\tau} x \frac{d y}{d x}=1+\frac{1+\tau}{\tau} \sum_{k \geq 1} \frac{\prod_{a=0}^{k-1}(k \tau+a)}{k !} x^{k} .
$$

We now define the polynomials

$$
\hat{\Psi}_{n}(t ; \tau)=\left(\left(\frac{t(t-1)(t \tau+1)}{\tau+1}\right) \frac{d}{d t}\right)^{n} \frac{t-1}{\tau+1}
$$

for $n \geq 0$. Finally, the Laplace transform of $\mathcal{C}_{g, \mu}(\tau)$ can be written in variable $t$,

$$
\begin{aligned}
\hat{\mathcal{C}}_{g, l}\left(t_{1}, . ., t_{l}\right) & =\sum_{\mu \in \mathbb{N}^{l}} \frac{1}{\sqrt{-1}^{l+|\mu|}} \mathcal{C}_{g}(\mu ; \tau) e^{-\left(\mu_{1} w_{1}+\cdots+\mu_{l} w_{l}\right)} \\
& =-(\tau(\tau+1))^{l-1} \sum_{\substack{b_{i} \geq 0 \\
i=1, . .,}}\left\langle\prod_{i=1}^{l} \tau_{b_{i}} \Gamma_{g}(\tau)\right\rangle_{g} \prod_{i=1}^{l} \hat{\Psi}_{b_{i}}\left(t_{i} ; \tau\right) .
\end{aligned}
$$

2.2. The Laplace transform of the cut-and-join equation. Let us introduce the formal variables $p=\left(p_{1}, . ., p_{n}, ..\right)$, and define $p_{\mu}=p_{\mu_{1}} \cdots p_{\mu_{l(\mu)}}$ for a partition $\mu$. We also introduce the generating functions

$$
\mathcal{C}_{g, l}(\lambda, p ; \tau)=\sum_{\mu, l(\mu)=l} \frac{\mathcal{C}_{g}(\mu ; \tau)}{|A u t(\mu)|} p_{\mu} \lambda^{2 g-2+l},
$$




$$
\mathcal{C}(\lambda, p ; \tau)=\sum_{\substack{g \geq 0 \\ l \geq 1}} \mathcal{C}_{g, l}(\lambda, p ; \tau)
$$

In [11], C. Liu, K. Liu and J. Zhou proved that $\mathcal{C}(\lambda, p ; \tau)$ satisfies the following cut-and-join equation

$$
\frac{\partial \mathcal{C}}{\partial \tau}=\frac{\sqrt{-1}}{2} \lambda \sum_{i, j \geq 1}\left((i+j) p_{i} p_{j} \frac{\partial \mathcal{C}}{\partial p_{i+j}}+i j p_{i+j} \frac{\partial^{2} \mathcal{C}}{\partial p_{i} \partial p_{j}}+i j p_{i+j} \frac{\partial \mathcal{C}}{\partial p_{i}} \frac{\partial \mathcal{C}}{\partial p_{j}}\right) .
$$

For every choice of $g \geq 1$ and a partition $\mu$, the coefficient of $p_{\mu} \lambda^{2 g-2+l(\mu)}$ in the above formula is

(6) $\frac{\partial \mathcal{C}_{g}(\mu ; \tau)}{\partial \tau}=\sqrt{-1} \sum_{i<j}\left(\mu_{i}+\mu_{j}\right) \mathcal{C}_{g}\left(\left(\mu(\hat{i}, \hat{j}), \mu_{i}+\mu_{j}\right) ; \tau\right)$

$$
\left.+\frac{\sqrt{-1}}{2} \sum_{i=1}^{l} \sum_{\alpha+\beta=\mu_{i}} \alpha \beta\left(\mathcal{C}_{g-1}(\mu(\hat{i}) ; \tau)\right)+\sum_{\substack{g_{1}+g_{2}=g \\ \nu_{1} \amalg \nu_{2}=\mu(\alpha, \hat{i})}} \mathcal{C}_{g_{1}}\left(\nu_{1} ; \tau\right) \mathcal{C}_{g_{2}}\left(\nu_{2} ; \tau\right)\right) .
$$

Let us first calculate the Laplace transform of the cut-and-join equation when $l=1$.

Proposition 2.2. When $g \geq 1$ and $l=1$, the Laplace transform of the cut-andjoin equation is:

$$
\begin{aligned}
& -\sum_{b \geq 0}\left\langle\tau_{b} \frac{d}{d \tau} \Gamma_{g}(\tau)\right\rangle_{g} \hat{\Psi}_{b}(t ; \tau)-\sum_{b \geq 0}\left\langle\tau_{b} \Gamma_{g}(\tau)\right\rangle_{g}\left(\frac{d}{d \tau} \hat{\Psi}_{b}(t ; \tau)+\frac{1}{t \tau+1} \hat{\Psi}_{b+1}(t ; \tau)\right) \\
& =\frac{1}{2} \sum_{a_{1}, a_{2} \geq 0}\left(\begin{array}{c}
\left.(\tau(\tau+1))\left\langle\tau_{a_{1}} \tau_{a_{2}} \Gamma_{g-1}(\tau)\right\rangle_{g-1}-\sum_{\substack{g_{1}+g_{2}=g \\
g_{1}>0 \\
g_{2}>0}}\left\langle\tau_{a_{1}} \Gamma_{g_{1}}(\tau)\right\rangle_{g_{1}}\left\langle\tau_{a_{2}} \Gamma_{g_{2}}(\tau)\right\rangle_{g_{2}}\right) \\
\cdot \hat{\Psi}_{a_{1}+1}(t ; \tau) \hat{\Psi}_{a_{2}+1}(t ; \tau) .
\end{array}\right)
\end{aligned}
$$

Proof. When $l=1$, the formula (6) is reduced to

(7) $\frac{\partial \mathcal{C}_{g}(\mu ; \tau)}{\partial \tau}=\frac{\sqrt{-1}}{2} \sum_{\alpha+\beta=\mu} \alpha \beta\left(\mathcal{C}_{g-1}((\alpha, \beta) ; \tau)+\sum_{g_{1}+g_{2}=g} \mathcal{C}_{g_{1}}(\alpha ; \tau) \mathcal{C}_{g_{2}}(\beta ; \tau)\right)$.

It then follows that the Laplace transformation of LHS of formula (7) is

$$
\frac{\partial}{\partial \tau} \hat{\mathcal{C}}_{g, 1}(t ; \tau)=\frac{\partial t}{\partial \tau} \frac{\partial}{\partial t} \hat{\mathcal{C}}_{g, 1}(t ; \tau)+\frac{\partial}{\partial \tau} \hat{\mathcal{C}}_{g, 1}(t ; \tau),
$$


and the stable part of RHS of (7) becomes

$$
\begin{aligned}
& \frac{1}{2}(\tau(\tau+1)) \sum_{a_{1}, a_{2} \geq 0}\left\langle\tau_{a_{1}} \tau_{a_{2}} \Gamma_{g-1}(\tau)\right\rangle_{g-1} \hat{\Psi}_{a_{1}+1}(t ; \tau) \hat{\Psi}_{a_{2}+1}(t ; \tau) \\
& -\frac{1}{2} \sum_{\substack{g_{1}+g_{2}=g \\
g_{1}>0 \\
g_{2}>0}}\left\langle\tau_{a_{1}} \Gamma_{g_{1}}(\tau)\right\rangle_{g_{1}}\left\langle\tau_{a_{2}} \Gamma_{g_{2}}(\tau)\right\rangle_{g_{2}} \hat{\Psi}_{a_{1}+1}(t ; \tau) \hat{\Psi}_{a_{2}+1}(t ; \tau),
\end{aligned}
$$

In the following, we calculate the Laplace transform of the unstable term $\mathcal{C}_{0}(\alpha ; \tau) \mathcal{C}_{g}(\beta ; \tau)+\mathcal{C}_{g}(\alpha ; \tau) \mathcal{C}_{0}(\beta ; \tau)$. Since $\mathcal{C}_{0, d}(\tau)=-\sqrt{-1}^{d+1} \frac{1}{\tau} \frac{\prod_{a=0}^{d-1}(d \tau+a)}{d !} \frac{1}{d^{2}}$, it follows that its Laplace transform $\hat{\mathcal{C}}_{0,1}(w ; \tau)=\sum_{d \geq 1} \frac{1}{\sqrt{-1}^{d+1}} \mathcal{C}_{0, d}(\tau) e^{-d w}$ satisfies:

$$
\left(-\frac{d}{d w}\right)^{2} \hat{\mathcal{C}}_{0,1}(w ; \tau)=-\frac{t-1}{\tau+1}=-\frac{y}{1-(\tau+1) y} .
$$

Hence $\frac{d}{d w} \hat{\mathcal{C}}_{0,1}(w ; \tau)=-\ln (1-y)$ followed by $-\frac{d}{d w}=\frac{(1-y) y}{1-(1+\tau) y} \frac{d}{d y}$. Moreover, recall that the framed Lambert curve is $y(1-y)^{\tau}=x$, where y depends on $\tau$. Taking the derivative of $\tau$, we have the identity

$$
-\ln (1-y)=\frac{\partial y}{\partial \tau} \frac{1-(\tau+1) y}{y(1-y)} .
$$

Therefore, the Laplace transform of the unstable part of RHS of (6) is

$$
\frac{\partial y}{\partial \tau} \frac{1-(\tau+1) y}{y(1-y)} \frac{\left(t^{2}-t\right)(t \tau+1)}{\tau+1} \frac{\partial}{\partial t} \hat{\mathcal{C}}_{g, 1}(t ; \tau)=\frac{\partial y}{\partial \tau} t^{2}(\tau+1) \frac{\partial}{\partial t} \hat{\mathcal{C}}_{g, 1}(t ; \tau)
$$

where we have used $t=\frac{1}{1-(\tau+1) y}$. We also have

$$
\frac{\partial t}{\partial \tau}=t^{2} y+\frac{\partial y}{\partial \tau} t^{2}(\tau+1)=\frac{t^{2}-t}{\tau+1}+\frac{\partial y}{\partial \tau} t^{2}(\tau+1) .
$$

Hence, moving the unstable part to left hand side of formula (7), then by formulas (8),(9) and (10), we obtain

$$
\left(\frac{\partial}{\partial \tau}+\frac{t^{2}-t}{(\tau+1)} \frac{\partial}{\partial t}\right) \hat{C}_{g, 1}(t ; \tau)=\text { stable part }
$$

which is the Proposition 2.2.

To finish the computation for $l \geq 2$, we introduce two lemmas firstly. 
Lemma 2.3. When $(g, l)=(0,2)$, we have the following Laplace transformation formula:

$$
\begin{aligned}
\hat{\mathcal{C}}_{0,2}\left(w_{1}, w_{2} ; \tau\right) & =-\sum_{\alpha, \beta \geq 1} \frac{\tau+1}{\tau} \frac{1}{\alpha+\beta} \frac{\prod_{a=0}^{\alpha-1}(\alpha \tau+a)}{\alpha !} \frac{\prod_{a=0}^{\beta-1}(\beta \tau+a)}{\beta !} e^{-\alpha w_{1}} e^{-\beta w_{2}} \\
& =-\ln \left(\frac{y_{1}-y_{2}}{x_{1}-x_{2}}\right)-\tau\left(\ln \left(1-y_{1}\right)+\ln \left(1-y_{2}\right)\right) .
\end{aligned}
$$

Proof. By definition,

$$
\begin{aligned}
\hat{\mathcal{C}}_{0,2}\left(w_{1}, w_{2} ; \tau\right) & =\sum_{\alpha, \beta \geq 1} \frac{1}{\sqrt{-1}^{2+\alpha+\beta}}|\operatorname{Aut}(\alpha, \beta)| \mathcal{C}_{0,(\alpha, \beta)}(\tau) \\
& =-\sum_{\alpha, \beta \geq 1} \frac{\tau+1}{\tau} \frac{1}{\alpha+\beta} \frac{\prod_{a=0}^{\alpha-1}(\alpha \tau+a)}{\alpha !} \frac{\prod_{a=0}^{\beta-1}(\beta \tau+a)}{\beta !} e^{-\alpha w_{1}} e^{-\beta w_{2}},
\end{aligned}
$$

it follows that

$$
\begin{aligned}
\left(-\frac{d}{d w_{1}}-\frac{d}{d w_{2}}\right) \hat{\mathcal{C}}_{0,2}\left(w_{1}, w_{2} ; \tau\right) & =-\tau(\tau+1) \hat{\Psi}_{0}\left(t_{1} ; \tau\right) \hat{\Psi}_{0}\left(t_{2} ; \tau\right) \\
& =-\tau(\tau+1) \frac{y_{1} y_{2}}{\left(1-(\tau+1) y_{1}\right)\left(1-(\tau+1) y_{2}\right)}
\end{aligned}
$$

Then, we obtain

$$
\hat{\mathcal{C}}_{0,2}\left(w_{1}, w_{2} ; \tau\right)=-\ln \left(\frac{y_{1}-y_{2}}{x_{1}-x_{2}}\right)-\tau\left(\ln \left(1-y_{1}\right)+\ln \left(1-y_{2}\right)\right)
$$

which followed by the relation

$$
-\frac{d}{d w}=x \frac{d}{d x}=\frac{(1-y) y}{1-(\tau+1) y} \frac{d}{d y} .
$$

Lemma 2.4. We have the following identity:

$$
\begin{aligned}
& \sum_{\alpha, \beta \geq 1} \frac{1}{\tau} \frac{\prod_{a=0}^{(\alpha+\beta)-1}((\alpha+\beta) \tau+a)}{(\alpha+\beta) !}(\alpha+\beta)^{a+1} e^{-\alpha w_{i}} e^{-\beta w_{j}} \\
& =\frac{x_{i}}{x_{i}-x_{j}} \hat{\Psi}_{a+1}\left(t_{i} ; \tau\right)-\frac{x_{j}}{x_{i}-x_{j}} \hat{\Psi}_{a+1}\left(t_{j} ; \tau\right)-\hat{\Psi}_{a+1}\left(t_{i} ; \tau\right)-\hat{\Psi}_{a+1}\left(t_{j} ; \tau\right) .
\end{aligned}
$$

Proof. This calculation is same as formula (3.15) showed in paper [7]. 
Let $\mu=\alpha+\beta$ and $\nu=\beta$, then

$$
\begin{aligned}
& \sum_{\alpha, \beta \geq 1} \frac{1}{\tau} \frac{\prod_{a=0}^{(\alpha+\beta)-1}((\alpha+\beta) \tau+a)}{(\alpha+\beta) !}(\alpha+\beta)^{a+1} e^{-\alpha w_{i}} e^{-\beta w_{j}} \\
& =\sum_{\alpha, \beta \geq 0} \frac{1}{\tau} \frac{\prod_{a=0}^{(\alpha+\beta)-1}((\alpha+\beta) \tau+a)}{(\alpha+\beta) !}(\alpha+\beta)^{a+1} e^{-\alpha w_{i}} e^{-\beta w_{j}} \\
& -\sum_{\alpha \geq 1} \frac{1}{\tau} \frac{\prod_{a=0}^{\alpha-1}(\alpha \tau+a)}{\alpha !} \alpha^{a+1} e^{-\alpha w_{i}}-\sum_{\beta \geq 1} \frac{1}{\tau} \frac{\prod_{a=0}^{\beta-1}(\beta \tau+a)}{\beta !} \beta^{a+1} e^{-\beta w_{j}} \\
& =\sum_{\mu \geq 0} \sum_{\nu=0}^{\mu} \frac{1}{\tau} \frac{\prod_{a=0}^{\mu-1}(\mu \tau+a)}{\mu !} \mu^{a+1} e^{-(\mu-\nu) w_{i}} e^{-\nu w_{j}}-\hat{\Psi}_{a+1}\left(t_{i} ; \tau\right)-\hat{\Psi}_{a+1}\left(t_{j} ; \tau\right) \\
& =\sum_{\mu \geq 0} \frac{1}{\tau} \frac{\prod_{a=0}^{\mu-1}(\mu \tau+a)}{\mu !} \mu^{a+1} \frac{x_{i}^{\mu+1}-x_{j}^{\mu+1}}{x_{i}-x_{j}}-\hat{\Psi}_{a+1}\left(t_{i} ; \tau\right)-\hat{\Psi}_{a+1}\left(t_{j} ; \tau\right) \\
& =\frac{x_{i}}{x_{i}-x_{j}} \hat{\Psi}_{a+1}\left(t_{i} ; \tau\right)-\frac{x_{j}}{x_{i}-x_{j}} \hat{\Psi}_{a+1}\left(t_{j} ; \tau\right)-\hat{\Psi}_{a+1}\left(t_{i} ; \tau\right)-\hat{\Psi}_{a+1}\left(t_{j} ; \tau\right) .
\end{aligned}
$$

Now we present our main result in this paper.

Theorem 2.5. For $g \geq 1$ and $l \geq 1$, we have the following equation:

$$
\begin{aligned}
(11) & -\left(\tau^{2}+\tau\right)^{l-2} \sum_{b_{L} \geq 0}\left((l-1)(2 \tau+1)\left\langle\tau_{b_{L}} \Gamma_{g}(\tau)\right\rangle_{g}+\left(\tau^{2}+\tau\right)\left\langle\tau_{b_{L}} \frac{d}{d \tau} \Gamma_{g}(\tau)\right\rangle_{g}\right) \\
& \cdot \hat{\Psi}_{b_{L}}\left(t_{L} ; \tau\right)-\left(\tau^{2}+\tau\right)^{l-1} \sum_{b_{L} \geq 0}\left\langle\tau_{b_{L}} \Gamma_{g}(\tau)\right\rangle_{g} \\
& \cdot \sum_{i=1}^{l}\left(\frac{\partial}{\partial \tau} \hat{\Psi}_{b_{i}}\left(t_{i} ; \tau\right)+\frac{1}{t_{i} \tau+1} \hat{\Psi}_{b_{i}+1}\left(t_{i} ; \tau\right)\right) \hat{\Psi}_{b_{L \backslash\{i\}}}\left(t_{L \backslash\{i\}} ; \tau\right) \\
= & -\frac{\left(\tau^{2}+\tau\right)^{l-2}}{\tau+1} \sum_{1 \leq i<j \leq l} \sum_{a \geq 0}\left\langle\tau_{a} \tau_{b_{L} \backslash\{i, j\}} \Gamma_{g}(\tau)\right\rangle_{g} \hat{\Psi}_{b_{L \backslash\{i, j\}}}\left(t_{L \backslash\{i, j\}} ; \tau\right) \\
& \cdot \frac{\left(t_{j}-1\right)\left(t_{i}^{2} \tau+t_{i}\right) \hat{\Psi}_{a+1}\left(t_{i} ; \tau\right)-\left(t_{i}-1\right)\left(t_{j}^{2} \tau+t_{j}\right) \hat{\Psi}_{a+1}\left(t_{j} ; \tau\right)}{t_{i}-t_{j}} \\
+ & \frac{\left(\tau^{2}+\tau\right)^{l-1}}{2} \sum_{i=1}^{l} \sum_{\substack{a_{1} \geq 0 \\
a_{2} \geq 0 \\
b_{L \backslash\{i\}} \geq 0}}\left(\left(\tau^{2}+\tau\right)\left\langle\tau_{a_{1}} \tau_{a_{2}} \tau_{b_{L} \backslash\{i\}} \Gamma_{g-1}(\tau)\right\rangle_{g-1}\right.
\end{aligned}
$$




$$
\begin{aligned}
& \left.-\sum_{\substack{g_{1}+g_{2}=g \\
\mathcal{I} \amalg \mathcal{J}=L \backslash\{i\} \\
2 g_{1}-1+|\mathcal{I}|>0 \\
2 g_{2}-1+|\mathcal{J}|>0}}\left\langle\tau_{a_{1}} \tau_{b_{\mathcal{I}}} \Gamma_{g_{1}}(\tau)\right\rangle_{g_{1}}\left\langle\tau_{a_{2}} \tau_{b_{\mathcal{J}}} \Gamma_{g_{2}}(\tau)\right\rangle_{g_{2}}\right) \\
& \cdot \prod_{n=1}^{2} \hat{\Psi}_{a_{n}+1}\left(t_{i} ; \tau\right) \hat{\Psi}_{b_{L \backslash\{i\}}}\left(t_{L \backslash\{i\}} ; \tau\right) .
\end{aligned}
$$

Proof. By definition, the Laplace transform of LHS of equation (6) is

$$
\begin{aligned}
& \sum_{\mu \in \mathbb{N}^{l}} \frac{1}{\sqrt{-1} l(\mu)+|\mu|} \frac{\partial}{\partial \tau} \mathcal{C}_{g}(\mu ; \tau) e^{-\left(\mu_{1} w_{1}+\cdots+\mu_{l} w_{l}\right)} \\
& \left.=\frac{\partial}{\partial \tau}\left(\sum_{\mu \in \mathbb{N}} \frac{1}{\sqrt{-1}} \mathcal{C}_{g}(\mu)+\mid \mu\right) e^{-\left(\mu_{1} w_{1}+\cdots+\mu_{l} w_{l}\right)}\right) \\
& =\frac{\partial}{\partial \tau} \hat{\mathcal{C}}_{g, l}\left(t_{1}, . ., t_{l} ; \tau\right) \\
& =\sum_{i=1}^{l} \frac{\partial t_{i}}{\partial \tau} \frac{\partial}{\partial t_{i}} \hat{\mathcal{C}}_{g, l}\left(t_{1}, . ., t_{l} ; \tau\right)+\frac{\partial}{\partial \tau} \hat{\mathcal{C}}_{g, l}\left(t_{1}, . ., t_{l} ; \tau\right) .
\end{aligned}
$$

The Laplace transform of stable geometry in the cut-term of RHS of (6) is

$$
\begin{aligned}
& \frac{\sqrt{-1}}{2} \sum_{\mu \in \mathbb{N}^{l}} \frac{1}{\sqrt{-1}^{l(\mu)+|\mu|}} \sum_{i=1}^{l} \sum_{\alpha+\beta=\mu_{i}} \alpha \beta\left(\mathcal{C}_{g-1}(\mu(\alpha, \hat{i}) ; \tau)\right. \\
& \left.+\sum_{\substack{g_{1}+g_{2}=g \\
\nu_{1} \amalg \nu_{2}=\mu(\alpha, \hat{i}) \\
2 g_{1}-2+\left|\nu_{1}\right|>0 \\
2 g_{2}-2+\left|\nu_{2}\right|>0}} \mathcal{C}_{g_{1}}\left(\nu_{1} ; \tau\right) \mathcal{C}_{g_{2}}(\nu ; \tau)\right) e^{-\left(\mu_{1} w_{1}+\cdots+\mu_{l} w_{l}\right)} \\
& =\frac{\left(\tau^{2}+\tau\right)^{l-1}}{2} \sum_{\substack { i=1 \\
\begin{subarray}{c}{a_{1} \geq 0 \\
a_{2} \geq 0 \\
b_{L \backslash\{i\}} \geq 0{ i = 1 \\
\begin{subarray} { c } { a _ { 1 } \geq 0 \\
a _ { 2 } \geq 0 \\
b _ { L \backslash \{ i \} } \geq 0 } }\end{subarray}}\left(\left(\tau^{2}+\tau\right)\left\langle\tau_{a_{1}} \tau_{a_{2}} \tau_{b_{L} \backslash\{i\}} \Gamma_{g-1}(\tau)\right\rangle_{g-1}\right.
\end{aligned}
$$




$$
\begin{aligned}
& \left.-\sum_{\substack{g_{1}+g_{2}=g \\
\mathcal{I} \amalg \mathcal{J}=\{1, ., \hat{i}, l l\} \\
2 g_{1}-1+\left|\mathcal{I}>0 \\
2 g_{2}-1+\right| \mathcal{J} \mid>0}}\left\langle\tau_{a_{1}} \tau_{b_{\mathcal{I}}} \Gamma_{g_{1}}(\tau)\right\rangle_{g_{1}}\left\langle\tau_{a_{2}} \tau_{b_{\mathcal{J}}} \Gamma_{g_{2}}(\tau)\right\rangle_{g_{2}}\right) \\
& \cdot \prod_{n=1}^{2} \hat{\Psi}_{a_{n}+1}\left(t_{i} ; \tau\right) \hat{\Psi}_{b_{L \backslash\{i\}}}\left(t_{L \backslash\{i\}} ; \tau\right) .
\end{aligned}
$$

The unstable geometry in the cut-term of RHS has two terms for $l \geq 2$ :

$$
\begin{gathered}
U_{1}=\frac{\sqrt{-1}}{2} \sum_{i=1}^{l} \sum_{\alpha+\beta=\mu_{i}} \alpha \beta\left(\mathcal{C}_{0}(\alpha ; \tau) \cdot \mathcal{C}_{g}((\mu(\hat{i}), \beta) ; \tau)+\mathcal{C}_{g}((\mu(\hat{i}), \alpha) ; \tau) \cdot \mathcal{C}_{0}(\beta ; \tau)\right), \\
U_{2}=\frac{\sqrt{-1}}{2} \sum_{i=1}^{l} \sum_{\alpha+\beta=\mu_{i}} \alpha \beta \sum_{j \neq i}\left(\mathcal{C}_{0}\left(\left(\mu_{j}, \alpha\right) ; \tau\right) \cdot \mathcal{C}_{g}((\mu(\hat{i}, \hat{j}), \beta) ; \tau)\right. \\
\quad+\mathcal{C}_{g}\left((\mu(\hat{i}, \hat{j}, \alpha) ; \tau) \cdot \mathcal{C}_{0}\left(\left(\mu_{j}, \beta\right) ; \tau\right)\right) .
\end{gathered}
$$

As calculated in the proof of Proposition 2.2, the Laplace transform of $U_{1}$ is

$$
\sum_{i=1}^{l} \frac{\partial y_{i}}{\partial \tau} t_{i}^{2}(\tau+1) \frac{\partial}{\partial t_{i}} \hat{\mathcal{C}}_{g, l}\left(t_{1}, . ., t_{l} ; \tau\right) .
$$

Moving the formula (14) to left hand side of (6), it will cancel the first term of formula (12), i.e.

$$
\begin{aligned}
& (12)-(14)=\left(\frac{\partial}{\partial \tau}+\sum_{i=1}^{l} \frac{t_{i}^{2}-t_{i}}{\tau+1} \frac{\partial}{\partial t_{i}}\right) \hat{\mathcal{C}}_{g, l}\left(t_{1}, . ., t_{l} ; \tau\right) \\
& =-\left(\tau^{2}+\tau\right)^{l-2} \sum_{b_{L} \geq 0}\left((l-1)(2 \tau+1)\left\langle\tau_{b_{L}} \Gamma_{g}(\tau)\right\rangle_{g}\right. \\
& \left.+\left(\tau^{2}+\tau\right)\left\langle\tau_{b_{L}} \frac{d}{d \tau} \Gamma_{g}(\tau)\right\rangle_{g}\right) \hat{\Psi}_{b_{L}}\left(t_{L} ; \tau\right)-\left(\tau^{2}+\tau\right)^{l-1} \sum_{b_{L} \geq 0}\left\langle\tau_{b_{L}} \Gamma_{g}(\tau)\right\rangle_{g} \\
& \cdot \sum_{i=1}^{l}\left(\frac{\partial}{\partial \tau} \hat{\Psi}_{b_{i}}\left(t_{i} ; \tau\right)+\frac{1}{t_{i} \tau+1} \hat{\Psi}_{b_{i}+1}\left(t_{i} ; \tau\right)\right) \hat{\Psi}_{b_{L \backslash\{i\}}}\left(t_{L \backslash\{i\}} ; \tau\right) .
\end{aligned}
$$

Furthermore, the Laplace transform of $U_{2}$ equals to

$$
\sum_{\mu \in \mathbb{N}^{l}} \frac{1}{\sqrt{-1}^{l+|\mu|}} U_{2} e^{-\left(\mu_{1} w_{1}+\cdots+\mu_{l} w_{l}\right)}
$$




$$
\begin{aligned}
& =-\sum_{i=1}^{l} \sum_{\substack{j \neq i \\
\mu_{j} \geq 1 \\
\alpha \geq 1}} \frac{1}{\sqrt{-1}^{2+\mu_{j}+\alpha}} \alpha \mathcal{C}_{0}\left(\left(\mu_{j}, \alpha\right) ; \tau\right) e^{-\mu_{j} w_{j}} e^{-\alpha w_{i}} \\
& \times \sum_{\substack{\beta \geq 0 \\
\mu_{k} \geq 0 \\
k \neq i, j}} \frac{1}{\sqrt{-1}^{l+|\mu|-\alpha-1}} \beta \mathcal{C}_{g}((\mu(\hat{i}, \hat{j}), \beta) ; \tau) e^{-\beta w_{i}} e^{-\sum_{k \neq i, j} \mu_{k} w_{k}} \\
& =\sum_{i=1}^{l} \sum_{j \neq i} \frac{\partial}{\partial w_{i}} \hat{\mathcal{C}}_{0,2}\left(w_{i}, w_{j} ; \tau\right)\left(\tau^{2}+\tau\right)^{l-2} \\
& \cdot \sum_{\substack{a \geq 0 \\
b_{L \backslash\{i, j\}} \geq 0}}\left\langle\tau_{a} \tau_{b_{L \backslash\{i, j\}}} \Gamma_{g}(\tau)\right\rangle_{g} \hat{\Psi}_{a+1}\left(t_{i} ; \tau\right) \hat{\Psi}_{b_{L \backslash\{i, j\}}}\left(t_{L \backslash\{i, j\}} ; \tau\right) \\
& =\sum_{i=1}^{l} \sum_{j \neq i}\left(-\frac{y_{i}}{y_{i}-y_{j}}\left(1+\frac{\tau y_{j}}{1-(\tau+1) y_{i}}\right)+\frac{x_{i}}{x_{i}-x_{j}}\right) \\
& \cdot\left(\tau^{2}+\tau\right)^{l-2} \sum_{\substack{a \geq 0 \\
b_{L \backslash\{i, j\}} \geq 0}}\left\langle\tau_{a} \tau_{b_{L \backslash\{i, j\}}} \Gamma_{g}(\tau)\right\rangle_{g} \hat{\Psi}_{a+1}\left(t_{i} ; \tau\right) \hat{\Psi}_{b_{L \backslash\{i, j\}}}\left(t_{L \backslash\{i, j\}} ; \tau\right) \\
& =\left(\tau^{2}+\tau\right)^{l-2} \sum_{1 \leq i<j \leq l} \sum_{\substack{a \geq 0 \\
b_{L \backslash\{i, j\} \geq 0}}}\left\langle\tau_{a} \tau_{b_{L \backslash\{i, j\}}} \Gamma_{g}(\tau)\right\rangle_{g} \hat{\Psi}_{b_{L \backslash\{i, j\}}}\left(t_{L \backslash\{i, j\}} ; \tau\right) \\
& \cdot\left(\frac{x_{i}}{x_{i}-x_{j}} \hat{\Psi}_{a+1}\left(t_{i} ; \tau\right)-\frac{x_{j}}{x_{i}-x_{j}} \hat{\Psi}_{a+1}\left(t_{j} ; \tau\right)\right. \\
& -\frac{y_{i}}{y_{i}-y_{j}}\left(1+\frac{\tau y_{j}}{1-(\tau+1) y_{i}}\right) \hat{\Psi}_{a+1}\left(t_{i} ; \tau\right) \\
& \left.+\frac{y_{j}}{y_{i}-y_{j}}\left(1+\frac{\tau y_{i}}{1-(\tau+1) y_{j}}\right) \hat{\Psi}_{a+1}\left(t_{j} ; \tau\right)\right)
\end{aligned}
$$

where we have used the Lemma 2.4.

Finally, we calculate the Laplace transform of the join term in RHS of (6)

$$
\begin{aligned}
& \sum_{\mu \in \mathbb{N}^{l}} \sum_{i<j} \frac{\sqrt{-1}}{\sqrt{-1} l(\mu)+|\mu|}\left(\mu_{i}+\mu_{j}\right) \mathcal{C}_{g}(\mu(\hat{i}, \hat{j}) ; \tau) e^{-\mu_{i} w_{i}} e^{-\mu_{j} w_{j}} e^{-\sum_{k \neq i, j} \mu_{k} w_{k}} \\
& =-\left(\tau^{2}+\tau\right)^{l-2} \sum_{\mu \in \mathbb{N}^{l}} \sum_{i<j} \sum_{\substack{a \geq 0 \\
b_{L \backslash\{i, j\}} \geq 0}}\left\langle\tau_{a} \tau_{b_{L} \backslash\{i, j\}} \Gamma_{g}(\tau)\right\rangle_{g} \prod_{k \neq i, j} \frac{1}{\tau} \frac{\prod_{a=0}^{\mu_{k}-1}\left(\mu_{k} \tau+a\right)}{\mu_{k} !}
\end{aligned}
$$




$$
\begin{aligned}
& \cdot \mu_{k}^{b_{k}} e^{-\sum_{k \neq i, j} \mu_{k} w_{k}} \frac{1}{\tau} \frac{\prod_{a=0}^{\mu_{i}+\mu_{j}-1}\left(\left(\mu_{i}+\mu_{j}\right) \tau+a\right)}{\left(\mu_{i}+\mu_{j}\right) !}\left(\mu_{i}+\mu_{j}\right)^{a+1} e^{-\mu_{i} w_{i}} e^{-\mu_{j} w_{j}} \\
& =-\left(\tau^{2}+\tau\right)^{l-2} \sum_{1 \leq i<j \leq l} \sum_{\substack{a \geq 0 \\
b_{L \backslash\{i, j\} \geq 0}}}\left\langle\tau_{a} \tau_{b_{L \backslash\{i, j\}}} \Gamma_{g}(\tau)\right\rangle_{g} \hat{\Psi}_{b_{L \backslash\{i, j\}}}\left(t_{L \backslash\{i, j\}} ; \tau\right) \\
& \cdot\left(\frac{x_{i}}{x_{i}-x_{j}} \hat{\Psi}_{a+1}\left(t_{i} ; \tau\right)-\frac{x_{j}}{x_{i}-x_{j}} \hat{\Psi}_{a+1}\left(t_{j} ; \tau\right)-\hat{\Psi}_{a+1}\left(t_{i} ; \tau\right)-\hat{\Psi}_{a+1}\left(t_{j} ; \tau\right)\right) .
\end{aligned}
$$

Combining (16) and (17), we get

$$
\begin{aligned}
& (16)+(17) \\
& =-\frac{\left(\tau^{2}+\tau\right)^{l-2}}{\tau+1} \sum_{1 \leq i<j \leq l} \sum_{\substack{a \geq 0 \\
b_{L \backslash\{i, j\}} \geq 0}}\left\langle\tau_{a} \tau_{b_{L \backslash\{i, j\}}} \Gamma_{g}(\tau)\right\rangle_{g} \hat{\Psi}_{b_{L \backslash\{i, j\}}}\left(t_{L \backslash\{i, j\}} ; \tau\right) \\
& . \frac{\left(t_{j}-1\right)\left(t_{i}^{2} \tau+t_{i}\right) \hat{\Psi}_{a+1}\left(t_{i} ; \tau\right)-\left(t_{i}-1\right)\left(t_{j}^{2} \tau+t_{j}\right) \hat{\Psi}_{a+1}\left(t_{j} ; \tau\right)}{t_{i}-t_{j}} .
\end{aligned}
$$

Collecting the remainder equations (15),(16) and (18), we obtain the identity (11) of Theorem 2.5.

\section{Some Hodge integral IDENTities}

In this section, we will show how to use the formula (1) to obtain some Hodge integral identities.

3.1. Preliminary calculations. For convenience, let us recall the formula (1) first: for $g \geq 1$ and $l \geq 1$,

$$
\begin{aligned}
& \text { LHS }:=-\left(\tau^{2}+\tau\right)^{l-2} \sum_{b_{L} \geq 0}\left((l-1)(2 \tau+1)\left\langle\tau_{b_{L}} \Gamma_{g}(\tau)\right\rangle_{g}\right. \\
& \left.+\left(\tau^{2}+\tau\right)\left\langle\tau_{b_{L}} \frac{d}{d \tau} \Gamma_{g}(\tau)\right\rangle_{g}\right) \hat{\Psi}_{b_{L}}\left(t_{L} ; \tau\right)-\left(\tau^{2}+\tau\right)^{l-1} \sum_{b_{L} \geq 0}\left\langle\tau_{b_{L}} \Gamma_{g}(\tau)\right\rangle_{g} \\
& \cdot \sum_{i=1}^{l}\left(\frac{\partial}{\partial \tau} \hat{\Psi}_{b_{i}}\left(t_{i} ; \tau\right)+\frac{1}{t_{i} \tau+1} \hat{\Psi}_{b_{i}+1}\left(t_{i} ; \tau\right)\right) \hat{\Psi}_{b_{L} \backslash\{i\}}\left(t_{L \backslash\{i\}} ; \tau\right) \\
& =T_{1}+T_{2}+T_{3}
\end{aligned}
$$


where

$$
\begin{aligned}
& T_{1}:=-\frac{\left(\tau^{2}+\tau\right)^{l-2}}{\tau+1} \sum_{1 \leq i<j \leq l} \sum_{\substack{a \geq 0 \\
b_{L \backslash\{i, j\}} \geq 0}}\left\langle\tau_{a} \tau_{b_{L \backslash\{i, j\}}} \Gamma_{g}(\tau)\right\rangle_{g} \hat{\Psi}_{b_{L \backslash\{i, j\}}}\left(t_{L \backslash\{i, j\}} ; \tau\right) \\
& \cdot \frac{\left(t_{j}-1\right)\left(t_{i}^{2} \tau+t_{i}\right) \hat{\Psi}_{a+1}\left(t_{i} ; \tau\right)-\left(t_{i}-1\right)\left(t_{j}^{2} \tau+t_{j}\right) \hat{\Psi}_{a+1}\left(t_{j} ; \tau\right)}{t_{i}-t_{j}}, \\
& T_{2}:=\frac{\left(\tau^{2}+\tau\right)^{l}}{2} \sum_{i=1}^{l} \sum_{\substack{a_{1} \geq 0 \\
a_{2} \geq 0 \\
b_{L \backslash\{i\}} \geq 0}}\left\langle\tau_{a_{1}} \tau_{a_{2}} \tau_{b_{L \backslash\{i\}}} \Gamma_{g-1}(\tau)\right\rangle_{g-1} \\
& \cdot \prod_{n=1}^{2} \hat{\Psi}_{a_{n}+1}\left(t_{i} ; \tau\right) \hat{\Psi}_{b_{L \backslash\{i\}}}\left(t_{L \backslash\{i\}} ; \tau\right) \\
& T_{3}:=-\frac{\left(\tau^{2}+\tau\right)^{l-1}}{2} \sum_{i=1}^{l} \sum_{\substack{a_{1} \geq 0 \\
a_{2} \geq 0 \\
b_{L} \backslash\{i\}}} \sum_{\substack{g_{1}+g_{2}=g \\
2 g_{1}-1+\mid \mathcal{J}=L \backslash\left\{0 \\
2 g_{2}-1+|\mathcal{J}|>0\right.}}\left\langle\tau_{a_{1}} \tau_{b_{\mathcal{I}}} \Gamma_{g_{1}}(\tau)\right\rangle_{g_{1}}\left\langle\tau_{a_{2}} \tau_{b_{\mathcal{J}}} \Gamma_{g_{2}}(\tau)\right\rangle_{g_{2}} \\
& \cdot \prod_{n=1}^{2} \hat{\Psi}_{a_{n}+1}\left(t_{i} ; \tau\right) \hat{\Psi}_{b_{L \backslash\{i\}}}\left(t_{L \backslash\{i\}} ; \tau\right), \\
& \hat{\Psi}_{n}(t ; \tau)=\left(\frac{\left(t^{2}-t\right)(t \tau+1)}{\tau+1} \frac{d}{d t}\right)^{n}\left(\frac{t-1}{\tau+1}\right) \text { for } n \geq 0,
\end{aligned}
$$

and

$$
\Gamma_{g}(\tau)=\Lambda_{g}^{\vee}(1) \Lambda_{g}^{\vee}(-\tau-1) \Lambda_{g}^{\vee}(\tau) .
$$

One can expand $\hat{\Psi}_{n}(t ; \tau)$ and $\Gamma_{g}(\tau)$ as follow,

$$
\hat{\Psi}_{b}(t ; \tau)=\sum_{k=0}^{b} \frac{\tau^{k}}{(\tau+1)^{b+1}} \Psi_{b}^{k}(t)
$$

and

$$
\Gamma_{g}(\tau)=\sum_{m=0}^{2 g} \Lambda_{g}^{\vee}(1) a_{m}(\lambda) \tau^{m} .
$$

By the definition of $\Lambda_{g}^{\vee}(t)=\sum_{j=0}^{g}(-1)^{g-j} \lambda_{g-j} t^{j}$ and Mumford's relation $\Lambda_{g}^{\vee}(t) \Lambda_{g}^{\vee}(-t)=$ $(-1)^{g} t^{2 g}$, one can obtain the coefficients $a_{m}(\lambda)$ in (21). For example,

$$
a_{2 g}(\lambda)=(-1)^{g}, a_{2 g-1}(\lambda)=(-1)^{g} g, \cdots
$$




$$
\begin{aligned}
& a_{1}(\lambda)=\sum_{m=1}^{g} m \lambda_{g-m} \lambda_{g}-(-1)^{g} \lambda_{g-1} \Lambda_{g}^{\vee}(-1), \\
& a_{0}(\lambda)=(-1)^{g} \lambda_{g} \Lambda_{g}^{\vee}(-1) .
\end{aligned}
$$

On the other hand, by definition $\hat{\Psi}_{b+1}(t, \tau)=\left(t^{2}-t\right)\left(\frac{t \tau+1}{\tau+1}\right) \frac{d}{d t} \hat{\Psi}_{b}(t, \tau), \hat{\Psi}_{0}(t, \tau)=$ $\frac{t-1}{\tau+1}$, it follows that $\hat{\Psi}_{b+1}(t, \tau)$ has the expansion form (20). Moreover, we have

$$
\Psi_{b+1}^{k}(t)=\left(t^{3}-t^{2}\right) \frac{d}{d t} \Psi_{b}^{k-1}(t)+\left(t^{2}-t\right) \frac{d}{d t} \Psi_{b}^{k}(t), k=0, . ., b+1 .
$$

and all the $\Psi_{b}^{k}(t)$ can be calculated.

As an illustration, we calculate some cases which will be used in the following discussions. By formula (23),

$$
\begin{gathered}
\Psi_{b+1}^{b+1}(t)=\left(t^{3}-t^{2}\right) \frac{d}{d t} \Psi_{b}^{b}(t), \\
\Psi_{b+1}^{b}(t)=\left(t^{3}-t^{2}\right) \frac{d}{d t} \Psi_{b}^{b-1}(t)+\left(t^{2}-t\right) \frac{d}{d t} \Psi_{b}^{b}(t), \\
\Psi_{b+1}^{1}(t)=\left(t^{3}-t^{2}\right) \frac{d}{d t} \Psi_{b}^{0}(t)+\left(t^{2}-t\right) \frac{d}{d t} \Psi_{b}^{1}(t), \\
\Psi_{b+1}^{0}(t)=\left(t^{2}-t\right) \frac{d}{d t} \Psi_{b}^{0}(t) .
\end{gathered}
$$

It is clear that, the recursion relation for $\Psi_{b}^{b}(t)$ in $(24)$ is same as the definition of $\hat{\xi}_{b}(t)$ in [7] and they share the same initial. Hence $\Psi_{b}^{b}(t)=\hat{\xi}_{b}(t)$. We write $\Psi_{b}^{b}(t)$ as

$$
\Psi_{b}^{b}(t)=\sum_{i=b+1}^{2 b+1} f^{b}(b, i) t^{i}
$$

then all the $f^{b}(b, i)$ could be calculated from the recursion (24). For example, $f^{b}(b, 2 b+1)=(2 b-1) ! !, f^{b}(b, b+1)=(-1)^{b} b$ ! which are the coefficients used in the proof of DVV equation and $\lambda_{g}$ integral respectively[14, 5, 9]. One can also let

$$
\Psi_{b}^{b-1}(t)=\sum_{i=b}^{2 b} f^{b-1}(b, i) t^{i}
$$

and by (25), we obtain

$$
f^{b}(b+1, k)=(k-2) f^{b-1}(b, k-2)-(k-1) f^{b-1}(b, k-1)
$$




$$
+(k-1) f^{b}(b, k-1)-k f^{b}(b, k) .
$$

For example,

$$
\begin{aligned}
f^{b}(b+1,2 b+2) & =2 b f^{b-1}(b, 2 b)+(2 b+1) f^{b}(b, 2 b+1) \\
& =2 b f^{b-1}(b, 2 b)+(2 b+1) ! !
\end{aligned}
$$

it follows that

$$
f^{b-1}(b, 2 b)=\sum_{k=0}^{b-1} \frac{(2 b-2) ! !(2 k+1) ! !}{(2 k) ! !}
$$

Similarly,

$$
\begin{aligned}
f^{b}(b+1, b+1) & =-b f^{b+1}(b, b)-(b+1) f^{b}(b, b+1) \\
& =-b f^{b}(b, b)+(-1)^{b+1}(b+1) !
\end{aligned}
$$

hence

$$
f^{b-1}(b, b)=(-1)^{b} \frac{(b+1) !}{2} .
$$

Moreover, we let

$$
\Psi_{b}^{0}(t)=\sum_{i=1}^{b+1} f^{0}(b, i) y^{i}
$$

and we have $f^{0}(b+1, i)=(i-1) f^{0}(b, i-1)-i f^{0}(b, i)$ from (27). It is easy to show

$$
f^{0}(b, b+1)=b !
$$

Similarly, one can write $\Psi_{b}^{1}(t)$ as

$$
\Psi_{b}^{1}(t)=\sum_{j=2}^{b+2} f^{1}(b, j) t^{j}
$$

where

$$
\begin{aligned}
f^{1}(b+1, k) & =(k-2) f^{0}(b, k-2)-(k-1) f^{0}(b, k-1) \\
& +(k-1) f^{1}(b, k-1)-k f^{1}(b, k) .
\end{aligned}
$$

Hence,

$$
f^{1}(b+1, b+3)=(b+1) f^{0}(b, b+1)+(b+2) f^{1}(b, b+2),
$$


by initial value $f^{1}(1,3)=1$ and $f^{0}(b, b+1)=b$ !, it follows

$$
f^{1}(b, b+2)=(b+1) ! \sum_{k=2}^{b+1} \frac{1}{k} .
$$

Now, let us substitute (20) and (21) to (19), one gets

$$
\begin{aligned}
& L H S=-\sum_{b_{L} \geq 0} \sum_{0 \leq k_{L} \leq b_{L}} \sum_{m=0}^{2 g}\left\langle\tau_{b_{L}} \Lambda_{g}^{\vee}(1) a_{m}(\lambda)\right\rangle_{g} \Psi_{b_{L}}^{k_{L}}\left(t_{L}\right) \\
& \cdot \frac{\left(l-2+m+\left|k_{L}\right|-\left|b_{L}\right|\right) \tau^{\left|k_{L}\right|+l+m-1}+\left(l-1+m+\left|k_{L}\right|\right) \tau^{\left|k_{L}\right|+l+m-2}}{(\tau+1)^{\left|b_{L}\right|+2}} \\
& -\sum_{b_{L} \geq 0} \sum_{0 \leq k_{L} \leq b_{L}} \sum_{m=0}^{2 g}\left\langle\tau_{b_{L}} \Lambda_{g}^{\vee}(1) a_{m}(\lambda)\right\rangle_{g} \\
& \sum_{i=1}^{l}\left(t_{i}^{2}-t_{i}\right) \frac{\partial}{\partial t_{i}} \Psi_{b_{i}}^{k_{i}}\left(t_{i}\right) \Psi_{b_{L \backslash\{i\}}}^{k_{L}}\left(t_{L \backslash\{i\}}\right) \frac{\tau^{\left|b_{L}\right|+m+l-2}}{(\tau+1)^{\left|b_{L}\right|+2}},
\end{aligned}
$$

$$
\begin{aligned}
& T_{1}=-\sum_{1 \leq i<j \leq l} \sum_{\substack{a \geq 0 \\
b_{L \backslash\{i, j\}} \geq 0}} \sum_{m=0}^{2 g} \sum_{\substack{0 \leq k \leq a+1 \\
0 \leq k_{L \backslash\{i, j\}} \leq b_{L \backslash\{i, j\}}}}\left\langle\tau_{a} \tau_{b_{L \backslash\{i, j\}}} \Lambda_{g}^{\vee}(1) a_{m}(\lambda)\right\rangle_{g} \\
& \cdot \Psi_{b_{L \backslash\{i, j\}}}^{k_{L \backslash\{i, j\}}}\left(t_{L \backslash\{i, j\}}\right) \frac{\left(t_{j}-1\right) t_{i}^{2} \Psi_{a+1}^{k}\left(t_{i}\right)-\left(t_{i}-1\right) t_{j}^{2} \Psi_{a+1}^{k}\left(t_{j}\right)}{t_{i}-t_{j}} \frac{\tau^{\left|k_{L \backslash\{i, j\}}\right|+k+l+m-1}}{(\tau+1)^{\left|b_{L \backslash\{i, j\}}\right|+a+3}} \\
& -\sum_{1 \leq i<j \leq l} \sum_{\substack{a \geq 0 \\
b_{L \backslash\{i, j\}} \geq 0}} \sum_{m=0}^{2 g} \sum_{\substack{0 \leq k \leq a+1 \\
0 \leq k_{L \backslash\{i, j\}} \leq b_{L \backslash\{i, j\}}}}\left\langle\tau_{a} \tau_{b_{L \backslash\{i, j\}}} \Lambda_{g}^{\vee}(1) a_{m}(\lambda)\right\rangle_{g} \\
& \text {. } \Psi_{b_{L \backslash\{i, j\}}}^{k_{L \backslash i, j\}}}\left(t_{L \backslash\{i, j\}}\right) \frac{\left(t_{j}-1\right) t_{i} \Psi_{a+1}^{k}\left(t_{i}\right)-\left(t_{i}-1\right) t_{j} \Psi_{a+1}^{k}\left(t_{j}\right)}{t_{i}-t_{j}} \frac{\tau^{\left|k_{L \backslash\{i, j\}}\right|+k+l+m-2}}{(\tau+1)^{\left|b_{L \backslash\{i, j\}}\right|+a+3}}, \\
& \text { (38) } \quad T_{2}=\frac{1}{2} \sum_{i=1}^{l} \sum_{\substack{a_{1} \geq 0 \\
a_{2} \geq 0 \\
b_{L} \backslash\{i\} \geq 0}} \sum_{m=0}^{2(g-1)} \sum_{\substack{0 \leq n_{1} \leq a_{1}+1 \\
0 \leq n_{2} \leq a_{2}+1 \\
0 \leq k_{L} \backslash\{i\} \leq b_{L \backslash\{i\}}}}\left\langle\tau_{a_{1}} \tau_{a_{2}} \tau_{b_{L} \backslash\{i, j\}} \Lambda_{g-1}^{\vee}(1) a_{m}(\lambda)\right\rangle_{g-1} \\
& \cdot \Psi_{a_{1}+1}^{n_{1}}\left(t_{i}\right) \Psi_{a_{2}+1}^{n_{2}}\left(t_{i}\right) \Psi_{b_{L \backslash\{i\}}}^{k_{L \backslash i\}}}\left(t_{L \backslash\{i\}}\right) \frac{\tau^{\left|k_{L \backslash\{i\}}\right|+n_{1}+n_{2}+l+m}}{(\tau+1)^{a_{1}+a_{2}+\left|k_{L \backslash\{i\}}\right|+3}},
\end{aligned}
$$




$$
\begin{aligned}
& T_{3}=-\frac{1}{2} \sum_{i=1}^{l} \sum_{\substack{a_{1} \geq 0 \\
a_{2} \geq 0 \\
b_{L} \backslash\{i\} \geq 0}} \sum_{\substack{g_{1}+g_{2}=g \\
\mathcal{I} \cup \mathcal{J}=L \backslash\{i\}}} \sum_{\substack{0 \leq n_{1} \leq a_{1}+1 \\
2 g_{1}-1+|\mathcal{I}|>0 \\
2 n_{2} \leq a_{2}+1 \\
0 \leq m_{1} \leq 2 g_{1} \\
0 \leq m_{2} \leq 2 g_{2} \\
0 \leq k_{L}-1+|J|>0}}\left\langle\tau_{a_{1}} \tau_{b_{\mathcal{I}}} \Lambda_{g_{1}}^{\vee}(1) a_{m_{1}}(\lambda)\right\rangle_{g_{1}} \\
& \cdot\left\langle\tau_{a_{2}} \tau_{b_{\mathcal{J}}} \Lambda_{g_{2}}^{\vee}(1) a_{m_{2}}(\lambda)\right\rangle_{g_{2}} \Psi_{a_{1}+1}^{n_{1}}\left(t_{i}\right) \Psi_{a_{2}+1}^{n_{2}}\left(t_{i}\right) \\
& \cdot \Psi_{b_{L \backslash\{i\}}}^{k_{L \backslash\{i\}}}\left(t_{L \backslash\{i\}}\right) \frac{\tau^{\left|k_{L \backslash\{i\}}\right|+n_{1}+n_{2}+l+m_{1}+m_{2}-1}}{(\tau+1)^{a_{1}+a_{2}+\left|k_{L \backslash\{i\}}\right|+4}},
\end{aligned}
$$

where we have used the notation $\left|b_{L}\right|=\sum_{i=1}^{l} b_{i}$ in above formulas. We note that (36), (37), (38) and (39) are all the functions of $\tau$. If we expand them as the formal power series of $\tau$ at different points, we will get some identities of Hodge integrals after recollecting the coefficients of $\tau$ at both sides of the identity $(36)=(37)+(38)+(39)$.

3.2. Expansion of $\tau$ at $\infty$. For $b \geq 0$, one can expand at $\tau=\infty$ by

$$
\frac{\tau^{a}}{(\tau+1)^{b}}=\sum_{h \geq 0}\left(\begin{array}{c}
-b \\
h
\end{array}\right) \tau^{a-b-h}
$$

For example, the term containing $\tau$ in (36) is

$$
\frac{\tau^{\left|k_{L}\right|+l+m-1}}{(\tau+1)^{\left|b_{L}\right|+2}}=\sum_{h \geq 0}\left(\begin{array}{c}
-\left|b_{L}\right|-2 \\
h
\end{array}\right) \tau^{l+m-3+\left|k_{L}\right|-\left|b_{L}\right|-h} .
$$

It is easy to see that the largest degree of $\tau$ is $2 g+l-3$ in the formulas (36), (37), (38), (39). If $F(t, \tau) \in Q[t][[\tau]]$, we will use the notation $\left[\tau^{k}\right] F(t, \tau)$ to denote the coefficient of $\tau^{k}$ in $F(t, \tau)$. By $a_{2 g}=(-1)^{g}$ in (22) and some calculations, we obtain

$$
\begin{gathered}
{\left[\tau^{2 g+l-3}\right] L H S=(-1)^{g+1} \sum_{b_{L} \geq 0}\left\langle\tau_{b_{L}} \Lambda_{g}^{\vee}(1)\right\rangle\left((2 g-2+l) \Psi_{b_{L}}^{b_{L}}\left(t_{L}\right)\right.} \\
\left.+\sum_{i=1}^{l}\left(t_{i}^{2}-t_{i}\right) \frac{\partial}{\partial t} \Psi_{b_{i}}^{b_{i}}\left(t_{i}\right) \Psi_{b_{L \backslash\{i\}}}^{b_{L \backslash\{i\}}}\left(t_{L \backslash\{i\}}\right)\right) \\
{\left[\tau^{2 g+l-3}\right] T_{1}=(-1)^{g+1} \sum_{1 \leq i<j \leq l} \sum_{\substack{a \geq 0 \\
b_{L \backslash\{i, j\}} \geq 0}}\left\langle\tau_{a} \tau_{b_{L \backslash\{i, j\}}} \Lambda_{g}^{\vee}(1)\right\rangle_{g}}
\end{gathered}
$$


$(42)$

$$
\begin{gathered}
{\left[\tau^{2 g+l-3}\right] T_{2}=(-1)^{g+1} \frac{1}{2} \sum_{i=1}^{l} \sum_{\substack{a_{1} \geq 0 \\
a_{2} \geq 0 \\
b_{L \backslash\{i\}} \geq 0}}\left\langle\tau_{a_{1}} \tau_{a_{2}} \tau_{b_{L \backslash\{i\}}} \Lambda_{g-1}^{\vee}(1)\right\rangle_{g-1}} \\
\cdot \Psi_{a_{1}+1}^{a_{1}+1}\left(t_{i}\right) \Psi_{a_{2}+1}^{a_{2}+1}\left(t_{i}\right) \Psi_{b_{L \backslash\{i\}}}^{b_{L \backslash\{i\}}}\left(t_{L \backslash\{i\}}\right) \\
{\left[\tau^{2 g+l-3}\right] T_{3}=(-1)^{g+1} \frac{1}{2} \sum_{i=1}^{l} \sum_{\substack{a_{1} \geq 0 \\
a_{2} \geq 0 \\
b_{L \backslash\{i\}} \geq 0}} \sum_{\substack{\mathcal{I} \cup \mathcal{J}=L \backslash\{i\} \\
g_{1}+1+|\mathcal{I}|>0 \\
2 g_{2}-1+|\mathcal{J}|>0}}\left\langle\tau_{a_{1}} \tau_{b_{\mathcal{I}}} \Lambda_{g_{1}}^{\vee}(1)\right\rangle_{g_{1}}} \\
\cdot\left\langle\tau_{a_{2}} \tau_{b_{\mathcal{J}}} \Lambda_{g_{2}}^{\vee}(1)\right\rangle_{g_{2}} \prod_{n=1}^{2} \Psi_{a_{n}+1}^{a_{n}+1}\left(t_{i}\right) \Psi_{b_{L \backslash\{i\}}}^{b_{L} \backslash\{i\}}\left(t_{L \backslash\{i\}}\right)
\end{gathered}
$$

Since $\Psi_{b}^{b}(t)=\hat{\xi}_{b}(t)$, we obtain the following corollary from the identity $(40)=$ $(41)+(42)+(43)$

\section{Corollary 3.1.}

$$
\begin{aligned}
& \text { (44) } \sum_{b_{L} \geq 0}\left\langle\tau_{b_{L}} \Lambda_{g}^{\vee}(1)\right\rangle\left((2 g-2+l) \hat{\xi}_{b_{L}}\left(t_{L}\right)+\sum_{i=1}^{l}\left(t_{i}^{2}-t_{i}\right) \frac{\partial}{\partial t} \hat{\xi}_{b_{i}}\left(t_{i}\right) \hat{\xi}_{b_{L \backslash\{i\}}}\left(t_{L \backslash\{i\}}\right)\right) \\
& =\sum_{1 \leq i<j \leq l} \sum_{\substack{a \geq 0 \\
b_{L \backslash\{i, j\}} \geq 0}}\left\langle\tau_{a} \tau_{b_{L \backslash\{i, j\}}} \Lambda_{g}^{\vee}(1)\right\rangle_{g} \hat{\xi}_{b_{L \backslash\{i, j\}}}\left(t_{L \backslash i, j}\right) \\
& \cdot \frac{\left(t_{j}-1\right) t_{i}^{2} \hat{\xi}_{a+1}\left(t_{i}\right)-\left(t_{i}-1\right) t_{j}^{2} \hat{\xi}_{a+1}\left(t_{j}\right)}{t_{i}-t_{j}} \\
& +\frac{1}{2} \sum_{i=1}^{l} \sum_{\substack{a_{1} \geq 0 \\
a_{2} \geq 0 \\
b_{L \backslash\{i\}} \geq 0}}\left(\left\langle\tau_{a_{1}} \tau_{a_{2}} \tau_{b_{L \backslash\{i\}}} \Lambda_{g-1}^{\vee}(1)\right\rangle_{g-1}+\sum_{\substack{g_{1}+g_{2}=g \\
\mathcal{I} \cup L \backslash\{i\} \\
2 g_{1}-1+|\mathcal{I}|>0 \\
2 g_{2}-1+|\mathcal{J}|>0}}\left\langle\tau_{a_{1}} \tau_{b_{\mathcal{I}}} \Lambda_{g_{1}}^{\vee}(1)\right\rangle_{g_{1}}\right. \\
& \left.\cdot\left\langle\tau_{a_{2}} \tau_{b_{\mathcal{J}}} \Lambda_{g_{2}}^{\vee}(1)\right\rangle_{g_{2}}\right) \hat{\xi}_{a_{1}+1}\left(t_{i}\right) \hat{\xi}_{a_{2}+1}\left(t_{i}\right) \hat{\xi}_{b_{L \backslash\{i\}}}\left(t_{L \backslash\{i\}}\right)
\end{aligned}
$$


which is Theorem 1.1, one of main results, in the paper [7]. Formula (44) has been studied by many people to derive DVV equation $[5,14], \lambda_{g}$-conjecture $[9,14]$ and $\lambda_{g-1}$ Hodge integral recursion [18].

Obviously, we can obtain more such corollaries with the same procedure. For example, by $a_{2 g}=(-1)^{g}, a_{2 g-1}(\lambda)=(-1)^{g} g$ in (22), we have

$$
\begin{aligned}
& {\left[\tau^{2 g+l-4}\right] L H S=(-1)^{g+1}(2 g-3+l) \sum_{b_{L} \geq 0}\left\langle\tau_{b_{L}} \Lambda_{g}^{\vee}(1)\right\rangle_{g}} \\
& \cdot\left[\left(g-1-\left|b_{L}\right|\right) \Psi_{b_{L}}^{b_{L}}\left(t_{L}\right)+\sum_{i=1}^{l} \Psi_{b_{i}}^{b_{i}-1}\left(t_{i}\right) \Psi_{b_{L \backslash\{i\}}}^{b_{L \backslash\{i}}\left(t_{L \backslash\{i\}}\right)\right]+(-1)^{g+1} \\
& \cdot \sum_{b_{L} \geq 0}\left\langle\tau_{b_{L}} \Lambda_{g}^{\vee}(1)\right\rangle_{g}\left[\sum_{i=1}^{l}\left(t_{i}^{2}-t_{i}\right) \frac{\partial}{\partial t_{i}}\left(\left(g-\left|b_{L}\right|-2\right) \Psi_{b_{i}}^{b_{i}}\left(t_{i}\right)+\Psi_{b_{i}}^{b_{i}-1}\left(t_{i}\right)\right)\right. \\
& \left.\left(\Psi_{b_{L \backslash\{i\}}}^{b_{L \backslash\{i\}}}\left(t_{L \backslash\{i\}}\right)+\sum_{j \neq i} \Psi_{b_{j}}^{b_{j}-1}\left(t_{j}\right) \Psi_{b_{L \backslash\{i, j\}}}^{b_{L \backslash\{i, j\}}}\left(t_{L \backslash\{i, j\}}\right)\right)\right]
\end{aligned}
$$

(46)

$$
\begin{aligned}
& {\left[\tau^{2 g+l-4}\right] T_{1}=(-1)^{g+1} \sum_{1 \leq i<j \leq l} \sum_{\substack{a \geq 0 \\
b_{L \backslash\{i, j\}} \geq 0}}\left\langle\tau_{a} \tau_{b_{L \backslash\{i, j\}}} \Lambda_{g}^{\vee}(1)\right\rangle_{g} \Psi_{b_{L \backslash\{i, j\}}}^{b_{L \backslash\{i, j\}}}\left(t_{L \backslash\{i, j\}}\right)} \\
& \cdot\left(\frac{\left(t_{j}-1\right) t_{i}^{2}\left[\Psi_{a+1}^{a}\left(t_{i}\right)+\left(g-a-\left|b_{L \backslash\{i, j\}}\right|-3+\frac{1}{t_{i}}\right) \Psi_{a+1}^{a+1}\left(t_{i}\right)\right]}{t_{i}-t_{j}}\right. \\
& \left.-\frac{\left(t_{i}-1\right) t_{j}^{2}\left[\Psi_{a+1}^{a}\left(t_{j}\right)+\left(g-a-\left|b_{L \backslash\{i, j\}}\right|-3+\frac{1}{t_{j}}\right) \Psi_{a+1}^{a+1}\left(t_{j}\right)\right]}{t_{i}-t_{j}}\right)
\end{aligned}
$$

$$
\begin{aligned}
& {\left[\tau^{2 g+l-4}\right] T_{2}=(-1)^{g+1} \frac{1}{2} \sum_{i=1}^{l} \sum_{\substack{a_{1} \geq 0 \\
a_{2} \geq 0 \\
b_{L \backslash\{i\} \geq 0}\\
}}\left\langle\tau_{a_{1}} \tau_{a_{2}} \tau_{b_{L \backslash\{i\}}} \Lambda_{g-1}^{\vee}(1)\right\rangle_{g-1}} \\
& \cdot\left[\left(g-a_{1}-a_{2}-\left|b_{L \backslash\{i, j\}}\right|-4\right) \Psi_{a_{1}+1}^{a_{1}+1}\left(t_{i}\right) \Psi_{a_{2}+1}^{a_{2}+1}\left(t_{i}\right)\right. \\
& \left.+\Psi_{a_{1}}^{a_{1}+1}\left(t_{i}\right) \Psi_{a_{2}+1}^{a_{2}+1}\left(t_{i}\right)+\Psi_{a_{1}+1}^{a_{1}+1}\left(t_{i}\right) \Psi_{a_{2}+1}^{a_{2}}\left(t_{i}\right)\right] \Psi_{b_{L \backslash\{i\}}}^{b_{L \backslash i\}}}\left(t_{L \backslash\{i\}}\right)
\end{aligned}
$$




$$
\begin{aligned}
& +(-1)^{g+1} \frac{1}{2} \sum_{i=1}^{l} \sum_{\substack{a_{1} \geq 0 \\
a_{2} \geq 0 \\
b_{L \backslash\{i\}} \geq 0}}\left\langle\tau_{a_{1}} \tau_{a_{2}} \tau_{b_{L \backslash\{i\}}} \Lambda_{g-1}^{\vee}(1)\right\rangle_{g-1} \\
& \cdot \Psi_{a_{1}}^{a_{1}+1}\left(t_{i}\right) \Psi_{a_{2}+1}^{a_{2}+1}\left(t_{i}\right) \sum_{j \neq i} \Psi_{b_{j}}^{b_{j}-1}\left(t_{j}\right) \Psi_{b_{L \backslash\{i, j\}}}^{b_{L \backslash\{i, j\}}}\left(t_{L \backslash\{i, j\}}\right) \\
& {\left[\tau^{2 g+l-4}\right] T_{3}=(-1)^{g+1} \frac{1}{2} \sum_{i=1}^{l} \sum_{\substack{a_{1} \geq 0 \\
a_{2} \geq 0 \\
b_{L \backslash\{i\}} \geq 0}} \sum_{\substack{g_{1}+g_{2}=g \\
\mathcal{I} \cup \mathcal{J}=L \backslash\{i\}}}^{\text {stable }}\left\langle\tau_{a_{1}} \tau_{b_{\mathcal{I}}} \Lambda_{g_{1}}^{\vee}(1)\right\rangle_{g_{1}}} \\
& \cdot\left\langle\tau_{a_{2}} \tau_{b_{\mathcal{J}}} \Lambda_{g_{2}}^{\vee}(1)\right\rangle_{g_{2}}\left[\left(g-a_{1}-a_{2}-\left|b_{L \backslash\{i, j\}}\right|-4\right) \Psi_{a_{1}+1}^{a_{1}+1}\left(t_{i}\right) \Psi_{a_{2}+1}^{a_{2}+1}\left(t_{i}\right)\right. \\
& \left.+\Psi_{a_{1}+1}^{a_{1}}\left(t_{i}\right) \Psi_{a_{2}+1}^{a_{2}+1}\left(t_{i}\right)+\Psi_{a_{1}+1}^{a_{1}+1}\left(t_{i}\right) \Psi_{a_{2}+1}^{a_{2}}\left(t_{i}\right)\right] \Psi_{b_{L \backslash\{i\}}}^{b_{L \backslash\{i\}}}\left(t_{L \backslash\{i\}}\right) \\
& +(-1)^{g+1} \frac{1}{2} \sum_{i=1}^{l} \sum_{\substack{a_{1} \geq 0 \\
a_{2} \geq 0 \\
b_{L \backslash\{i\}} \geq 0}} \sum_{\substack{\mathcal{I} \cup \mathcal{J}=L \backslash\{i\} \\
g_{1}+g_{1}=g \\
2 g_{2}-1+|\mathcal{I}|>0}}\left\langle\tau_{a_{1}} \tau_{b_{\mathcal{I}}} \Lambda_{g_{1}}^{\vee}(1)\right\rangle_{g_{1}} \\
& \cdot\left\langle\tau_{a_{2}} \tau_{b_{\mathcal{J}}} \Lambda_{g_{2}}^{\vee}(1)\right\rangle_{g_{2}} \Psi_{a_{1}}^{a_{1}+1}\left(t_{i}\right) \Psi_{a_{2}+1}^{a_{2}+1}\left(t_{i}\right) \sum_{j \neq i} \Psi_{b_{j}}^{b_{j}-1}\left(t_{j}\right) \Psi_{b_{L \backslash\{i, j\}}}^{b_{L \backslash\{i, j\}}}\left(t_{L \backslash\{i, j\}}\right)
\end{aligned}
$$

Then, we also get a Hodge integral identity from the identity $(45)=(46)+$ $(47)+(48)$ which contains the Hodge integral of type $\left\langle\tau_{b_{L}} \Lambda_{g}^{\vee}(1)\right\rangle_{g}$.

In order to get the Hodge integral identity with more than one $\lambda$-class, we need to compare the coefficients of $\tau^{2 g+l-5}$ with the same procedure, we will obtain a Hodge integral identity which involves $\left\langle\tau_{b_{L}} \Lambda_{g}^{\vee}(1)\right\rangle_{g}$ and $\left\langle\tau_{b_{L}} \Lambda_{g}^{\vee}(1) \lambda_{1}\right\rangle_{g}$, because $a_{2 g-2}(\lambda)=(-1)^{g}\left(\frac{g(g-1)}{2}-\lambda_{1}\right)$. And by this Hodge integral identity, we can calculate all the Hodge integral of the type $\left\langle\tau_{b_{L}} \lambda_{k} \lambda_{1}\right\rangle_{g}, k=1, . ., g$. However, many terms will appear in the computation of the coefficients of $\tau^{2 g+l-5}$ which makes the computation very complicated. So far, we have not written down the explicit formula to calculate the Hodge integral of the type $\left\langle\tau_{b_{L}} \lambda_{k} \lambda_{1}\right\rangle_{g}$.

3.3. Expansion of $\tau$ at $\mathbf{0}$. Now, let us consider the expansion of $\frac{\tau^{a}}{(\tau+1)^{b}}$ at $\tau=0$,

$$
\frac{\tau^{a}}{(\tau+1)^{b}}=\sum_{i \geq 0}\left(\begin{array}{c}
-b \\
i
\end{array}\right) \tau^{a+i}
$$


It is easy to see that the lowest degree of $\tau$ in formula (36) is $l-2$. By $a_{0}(\lambda)=$ $(-1)^{g} \lambda_{g} \Lambda_{g}^{\vee}(-1)$ show in $(22)$ and $\Lambda_{g}^{\vee}(1) \Lambda_{g}^{\vee}(-1)=(-1)^{g}$, we have

$$
\begin{gathered}
{\left[\tau^{l-2}\right] T_{1}=-\sum_{1 \leq i<j \leq l} \sum_{\substack{a \geq 0 \\
b_{L \backslash\{i, j\}} \geq 0}}\left\langle\tau_{a} \tau_{b_{L \backslash\{i, j\}}} \lambda_{g}\right\rangle_{g} \Psi_{b_{L \backslash\{i, j\}}}^{0}\left(t_{L \backslash\{i, j\}}\right)} \\
\times \frac{\left(t_{j}-1\right) t_{i}^{2} \Psi_{a+1}^{0}\left(t_{i}\right)-\left(t_{i}-1\right) t_{j}^{2} \Psi_{a+1}^{0}\left(t_{j}\right)}{t_{i}-t_{j}} \\
{\left[\tau^{l-2}\right] T_{2}=\left[\tau^{l-2}\right] T_{3}=0 .}
\end{gathered}
$$

Therefore, we obtain

\section{Corollary 3.2.}

$$
\begin{aligned}
& \sum_{b_{L} \geq 0}\left\langle\tau_{b_{L}} \lambda_{g}\right\rangle_{g} \Psi_{b_{L}}^{0}\left(t_{L}\right)=\frac{1}{l-1} \sum_{1 \leq i<j \leq l} \sum_{\substack{a \geq 0 \\
b_{L \backslash\{i, j\} \geq 0}}}\left\langle\tau_{a} \tau_{b_{L \backslash\{i, j\}}} \lambda_{g}\right\rangle_{g} \\
& \cdot \Psi_{b_{L \backslash\{i, j\}}}^{0}\left(t_{L \backslash\{i, j\}}\right) \frac{\left(t_{j}-1\right) t_{i}^{2} \Psi_{a+1}^{0}\left(t_{i}\right)-\left(t_{i}-1\right) t_{j}^{2} \Psi_{a+1}^{0}\left(t_{j}\right)}{t_{i}-t_{j}} .
\end{aligned}
$$

We now introduce two notations for the following expositions. Let

$$
g\left(x_{1}, . ., x_{l}\right)=\sum_{i_{k} \geq 0} a_{i_{1} i_{2} \cdots i_{l}} x_{1}^{i_{1}} \cdots x_{l}^{i_{l}} \in Q\left[x_{1}, . ., x_{l}\right]
$$

and denote by

$$
F_{d}\left(g\left(x_{1}, \cdots, x_{l}\right)\right)=\sum_{\sum_{k=1}^{l} i_{k}=d} a_{i_{1} i_{2} \cdots i_{l}} x_{1}^{i_{1}} \cdots x_{l}^{i_{l}}
$$

and

$$
\left[x_{1}^{j_{1}} \cdots x_{l}^{j_{l}}\right] g\left(x_{1}, \cdots, x_{l}\right)=a_{j_{1} j_{2} \cdots j_{l}} .
$$

the two operators acting on $g\left(x_{1}, . ., x_{l}\right)$. Applying the above two operators on Corollary 3.2, we have

$$
F_{2 g-3+2 l}(L H S \text { of }(52))=(l-1) \sum_{b_{L} \geq 0}\left\langle\tau_{b_{L}} \lambda_{g}\right\rangle_{g} b_{L} ! y_{L}^{b_{L}+1},
$$




$$
\begin{aligned}
F_{2 g-3+2 l}(R H S \text { of }(52)) & =\sum_{1 \leq i<j \leq l} \sum_{\substack{a \geq 0 \\
b_{L} \geq 0}}\left\langle\tau_{b_{L \backslash\{i, j\}}} \lambda_{g}\right\rangle_{g}(a+1) ! \\
& \cdot b_{L \backslash\{i, j\}} ! t_{L \backslash\{i, j\}}^{b_{L \backslash i, j\}}+1} \frac{t_{j} t_{i}^{a+4}-t_{i} t_{j}^{a+4}}{t_{i}-t_{j}} .
\end{aligned}
$$

It follows that

$$
\begin{gathered}
{\left[t_{L}^{b_{L}+1}\right] F_{2 g-3+2 l}(\text { LHS of }(52))=(l-1)\left\langle\tau_{b_{L}} \lambda_{g}\right\rangle_{g} b_{L} !} \\
{\left[t_{L}^{b_{L}+1}\right] F_{2 g-3+2 l}(R H S \text { of }(52))=\sum_{1 \leq i<j \leq l}\left\langle\tau_{b_{i}+b_{j}-1} \tau_{b_{L} \backslash\{i, j\}} \lambda_{g}\right\rangle_{g}\left(b_{i}+b_{j}\right) ! b_{L \backslash\{i, j\}} !}
\end{gathered}
$$

So we get

$$
\left\langle\tau_{b_{L}} \lambda_{g}\right\rangle_{g}=\frac{1}{l-1} \sum_{1 \leq i<j \leq l}\left\langle\tau_{b_{i}+b_{j}-1} \tau_{b_{L \backslash\{i, j\}}} \lambda_{g}\right\rangle_{g} \frac{\left(b_{i}+b_{j}\right) !}{b_{i} ! b_{j} !} .
$$

By induction on $l$, it follows the $\lambda_{g}$-integral formula:

$$
\left\langle\tau_{b_{L}} \lambda_{g}\right\rangle_{g}=\left(\begin{array}{c}
2 g-3+l \\
b_{L}
\end{array}\right) c_{g}
$$

In the following, we calculate the sub-lowest degree of $\tau$. By $a_{0}(\lambda)=(-1)^{g} \lambda_{g} \Lambda_{g}^{\vee}(-1)$ and $\Lambda_{g}^{\vee}(1) \Lambda_{g}^{\vee}(-1)=(-1)^{g}$, we have

$$
\begin{aligned}
{\left[\tau^{l-1}\right] L H S } & =-\sum_{b_{L} \geq 0}\left\langle\tau_{b_{L}} \lambda_{g}\right\rangle_{g} l\left(-\left(\left|b_{L}\right|+1\right) \Psi_{b_{L}}^{0}\left(t_{L}\right)+\sum_{j=1} \Psi_{b_{j}}^{1}\left(t_{j}\right) \Psi_{b_{L \backslash\{j\}}}^{0}\left(t_{L \backslash\{j\}}\right)\right) \\
& -\sum_{b_{L} \geq 0}\left\langle\tau_{b_{L}} \lambda_{g}\right\rangle_{g} \sum_{i=1}^{l}\left(t_{i}^{2}-t_{i}\right) \frac{\partial}{\partial t_{i}} \Psi_{b_{i}}^{0}\left(t_{i}\right) \Psi_{b_{L \backslash\{i\}}}^{0}\left(t_{L \backslash\{i\}}\right) \\
& -\sum_{b_{L} \geq 0}\left\langle\tau_{b_{L}} \Lambda_{g}^{\vee}(1) a_{1}(\lambda)\right\rangle_{g} l \Psi_{b_{L}}^{0}\left(t_{L}\right),
\end{aligned}
$$

(54)

$$
\begin{aligned}
& {\left[\tau^{l-1}\right] T_{1}=-\sum_{1 \leq i<j \leq l} \sum_{\substack{a \geq 0 \\
b_{L \backslash\{i, j\}} \geq 0}}\left\langle\tau_{a} \tau_{b_{L \backslash\{i, j\}}} \lambda_{g}\right\rangle_{g}} \\
& \cdot\left[\frac{\left(t_{j}-1\right) t_{i}\left(\Psi_{a+1}^{1}\left(t_{i}\right)+\left(t_{i}-\left|b_{L \backslash\{i, j\}}\right|-a-3\right) \Psi_{a+1}^{0}\left(t_{i}\right)\right)}{t_{i}-t_{j}}\right.
\end{aligned}
$$




$$
\begin{aligned}
& \left.-\frac{\left(t_{i}-1\right) t_{j}\left(\Psi_{a+1}^{1}\left(t_{j}\right)+\left(t_{j}-\left|b_{L \backslash\{i, j\}}\right|-a-3\right) \Psi_{a+1}^{0}\left(t_{j}\right)\right)}{t_{i}-t_{j}}\right] \Psi_{b_{L \backslash\{i, j\}}}^{0}\left(t_{L \backslash\{i, j\}}\right) \\
& -\sum_{1 \leq i<j \leq l} \sum_{\substack{a \geq 0 \\
b_{L \backslash\{i, j\}} \geq 0}}\left\langle\tau_{a} \tau_{b_{L \backslash\{i, j\}}} \lambda_{g}\right\rangle_{g} \sum_{r \neq i, j} \Psi_{b_{r}}^{1}\left(t_{r}\right) \Psi_{b_{L \backslash\{i, j\}}}^{0}\left(t_{L \backslash\{i, j\}}\right) \\
& \cdot \frac{\left(t_{j}-1\right) t_{i} \Psi_{a+1}^{0}\left(t_{i}\right)-\left(t_{i}-1\right) t_{j} \Psi_{a+1}^{0}\left(t_{j}\right)}{t_{i}-t_{j}} \\
& -\sum_{1 \leq i<j \leq l} \sum_{a \geq 0}\left\langle\tau_{a} \tau_{b_{L \backslash\{i, j\}}} \Lambda_{g}^{\vee}(1) a_{1}(\lambda)\right\rangle_{g} \\
& \cdot \Psi_{b_{L \backslash\{i, j\}} \geq 0}^{0}\left(t_{L \backslash\{i, j\}}\right) \frac{\left(t_{j}-1\right) t_{i} \Psi_{a+1}^{0}\left(t_{i}\right)-\left(t_{i}-1\right) t_{j} \Psi_{a+1}^{0}\left(t_{j}\right)}{t_{i}-t_{j}},
\end{aligned}
$$

$$
\begin{gathered}
{\left[\tau^{l-1}\right] T_{2}=0,} \\
{\left[\tau^{l-1}\right] T_{3}=-\frac{1}{2} \sum_{i=1}^{l} \sum_{\substack{a_{1} \geq 0 \\
a_{2} \geq 0 \\
b_{L \backslash\{i\}} \geq 0}} \sum_{\substack{g_{1}+g_{2}=g \\
\mathcal{I} \cup \mathcal{J}=L \backslash\{i\} \\
2 g_{1}-1+|\mathcal{I}|>0 \\
2 g_{2}-1+|\mathcal{J}|>0}}\left\langle\tau_{a_{1}} \tau_{b_{\mathcal{I}}} \lambda_{g_{1}}\right\rangle_{g_{1}}\left\langle\tau_{a_{2}} \tau_{b_{\mathcal{J}}} \lambda_{g_{2}}\right\rangle_{g_{2}}} \\
\cdot \Psi_{a_{1}+1}^{0}\left(t_{i}\right) \Psi_{a_{2}+1}^{0}\left(t_{i}\right) \Psi_{b_{L \backslash\{i\}}}^{0}\left(t_{L \backslash\{i\}}\right) .
\end{gathered}
$$

Recall $a_{1}(\lambda)=\sum_{m=1}^{g} m \lambda_{g-m} \lambda_{g}-(-1)^{g} \Lambda_{g}^{\vee}(-1) \lambda_{g-1}$ in (22) and Mumford's relation $\Lambda_{g}^{\vee}(1) \Lambda_{g}^{\vee}(-1)=(-1)^{g}$. We have the form

$$
\Lambda_{g}^{\vee}(1) a_{1}(\lambda)=\sum_{d=g-1}^{3 g-3} P_{d}(\lambda)
$$

where $P_{d}(\lambda)$ is some combinatoric of $\lambda$-class with degree $d$. For example

$$
\begin{aligned}
& P_{g-1}(\lambda)=\lambda_{g-1}, P_{g}(\lambda)=g \lambda_{g}, P_{g+1}(\lambda)=-\lambda_{g} \lambda_{1}, \\
& \cdots, P_{3 g-3}(\lambda)=(-1)^{g+1} \lambda_{g} \lambda_{g-1} \lambda_{g-2} .
\end{aligned}
$$

By the above calculation, substituting (57) to the identity $(53)=(54)+(56)$, we obtain Corollary 1.4 as showed in Section 1.

Since $\left\langle\tau_{b_{L}} \lambda_{g}\right\rangle_{g}=\left(\begin{array}{c}2 g-3+l \\ b_{L}\end{array}\right) c_{g}$, thus all the Hodge integrals of type

$$
\left\langle\tau_{b_{L}} \sum_{d=g-1}^{3 g-3} P_{d}(\lambda)\right\rangle_{g}
$$


can be calculated by Corollary 1.4.

3.4. A recursion formula for Hodge integral $\left\langle\tau_{b_{L}} \lambda_{g} \lambda_{1}\right\rangle_{g}$. Although Corollary 1.4 says that all the Hodge integral (59) is computable, it is not easy to write down their explicit formula. In this subsection, we will show how to obtain an recursion formula for $\left\langle\tau_{b_{L}} \lambda_{g} \lambda_{1}\right\rangle_{g}$.

We have known that $\left\langle\tau_{b_{L}} \lambda_{g}\right\rangle_{g}=\left(\begin{array}{c}2 g-3+l \\ b_{L}\end{array}\right) c_{g}$, and $\left\langle\tau_{b_{L}} \lambda_{g-1}\right\rangle_{g}$ can also be calculated easily from a recursion formula showed in [18]. Thus, we have

Theorem 3.3. If $\sum_{i=1}^{l} b_{i}=2 g-4+l$, there exists a constant $C\left(g, l, b_{1}, . ., b_{l}\right)$ related to $g, l, b_{1}, . ., b_{l}$, such that

$$
\left\langle\tau_{b_{L}} \lambda_{g} \lambda_{1}\right\rangle_{g}=\frac{1}{l} \sum_{1 \leq i<j \leq l}\left\langle\tau_{b_{i}+b_{j}-1} \tau_{b_{L \backslash\{i, j\}}} \lambda_{g} \lambda_{1}\right\rangle_{g} \frac{\left(b_{i}+b_{j}\right) !}{b_{i} ! b_{j} !}+C\left(g, l, b_{1}, . . b_{l}\right)
$$

where $C\left(g, l, b_{1}, . ., b_{l}\right)$ is a combinatoric constant related to the Hodge integrals $\left\langle\tau_{b_{L}} \lambda_{g}\right\rangle_{g}=\left(\begin{array}{c}2 g-3+l \\ b_{L}\end{array}\right) c_{g}$, and $\left\langle\tau_{b_{L}} \lambda_{g-1}\right\rangle_{g}$.

Proof. Taking all the terms with degree $2 g+2 l-4$ in formula Corollary 1.4, we have

$$
\begin{gathered}
F_{2 g+2 l-4}(L H S)=-l \sum_{b_{L} \geq 0}\left\langle\tau_{b_{L}} \lambda_{g} \lambda_{1}\right\rangle_{g} b_{L} !_{L}^{b_{L}+1}+G_{1}\left(t_{1}, . ., t_{l}\right) \\
F_{2 g+2 l-4}(R H S)=-\sum_{1 \leq i<j \leq l} \sum_{\substack{a \geq 0 \\
b_{L \backslash\{i, j\}} \geq 0}}\left\langle\tau_{a} \tau_{b_{L \backslash\{i, j\}}} \lambda_{g} \lambda_{1}\right\rangle_{g} \\
\cdot b_{L \backslash\{i, j\}} !(a+1) ! \sum_{m=0}^{a+1} t_{L \backslash\{i, j\}}^{b_{L \backslash i, j}+1} t_{i}^{a+2-m} t_{j}^{m+1}+G_{2}\left(t_{1}, . ., t_{l}\right)
\end{gathered}
$$

where $G_{i}\left(t_{1}, . ., t_{l}\right)=\sum_{\left|d_{L}\right|=2 g+2 l-4} C_{i}\left(g, l, d_{1}, . ., d_{l}\right) t_{L}^{d_{L}} \in \mathbb{Q}\left[t_{1}, . . t_{l}\right], C_{i}\left(g, l, d_{1}, . ., d_{l}\right)$ is a combinatoric constant related to $g, l, d_{1}, . ., d_{l}$. It follows that

$$
\begin{aligned}
& \sum_{b_{L} \geq 0}\left\langle\tau_{b_{L}} \lambda_{g} \lambda_{1}\right\rangle_{g} b_{L} ! t_{L}^{b_{L}+1}=\frac{1}{l} \sum_{1 \leq i<j \leq l} \sum_{\substack{a \geq 0 \\
b_{L \backslash\{i, j\}} \geq 0}}\left\langle\tau_{a} \tau_{b_{L \backslash\{i, j\}}} \lambda_{g} \lambda_{1}\right\rangle_{g} \\
& \cdot b_{L \backslash\{i, j\}} !(a+1) ! \sum_{m=0}^{a+1} t_{L \backslash\{i, j\}}^{b_{L \backslash\{i, j\}}+1} t_{i}^{a+2-m} t_{j}^{m+1}+G\left(t_{1}, . ., t_{l}\right)
\end{aligned}
$$


where $G\left(t_{1}, . ., t_{l}\right)=\sum_{\left|d_{L}\right|=2 g+2 l-4} \hat{C}\left(g, l, b_{1}, . ., b_{l}\right) t_{L}^{d_{L}}=\frac{1}{l}\left(G_{1}\left(t_{1}, . . t_{l}\right)-G_{2}\left(t_{1}, . ., t_{l}\right)\right)$. Then taking the coefficients of $t_{L}^{b_{L}+1}$ in (63), we get

$$
\begin{aligned}
\left\langle\tau_{b_{L}} \lambda_{g} \lambda_{1}\right\rangle_{g} & =\frac{1}{l} \sum_{1 \leq i<j \leq l}\left\langle\tau_{b_{i}+b_{j}-1} \tau_{b_{L} \backslash\{i, j\}} \lambda_{g} \lambda_{1}\right\rangle_{g} \frac{\left(b_{i}+b_{j}\right) !}{b_{i} ! b_{j} !} \\
& +\frac{\hat{C}\left(g, l, b_{1}+1, . ., b_{l}+1\right)}{b_{L} !}
\end{aligned}
$$

Let $C\left(g, l, b_{1}, . ., b_{l}\right)=\frac{\hat{C}\left(g, l, b_{1}+1, . ., b_{l}+1\right)}{b_{L} !}$, Theorem 3.4 is proved.

The initial value $\left\langle\tau_{2 g-3} \lambda_{g} \lambda_{1}\right\rangle_{g}=\frac{1}{12}\left[g(2 g-3) b_{g}+b_{1} b_{g-1}\right]$ has been obtained by Y. Li in [10]. Therefore, by the above recursion formula (64), one can calculate all the Hodge integral $\left\langle\tau_{b_{L}} \lambda_{g} \lambda_{1}\right\rangle_{g}$. This Hodge integral can also be computed using Mumford's Chern character formula for $\lambda_{1}$ and the $\lambda_{g}$ theorem [15].

\section{Conclusion}

With the help of the Laplace transform in this paper or the symmetrization method in [3], we can convert the cut-and-join equation of Mariño-Vafa formula to a polynomial identity which can be used to prove the BKMP conjecture [1] for $\mathbb{C}^{3}[4,17]$. In this paper, we derived some corollaries about the Hodge integral identities from this polynomial identity. In fact, we have found an algorithm to calculate the Hodge integrals appearing in Mariño-Vafa formula by the following two steps.

Step1: expanding $\tau$ at special points, then collect the corresponding level of $\tau$ in Theorem 1.1.

Step2: taking certain degrees of $t_{L}$ to obtain the corresponding Hodge integral identities.

In this paper, we only calculate four cases by using Step1 and calculate a new Hodge integral recursion for $\left\langle\tau_{b_{L}} \lambda_{g} \lambda_{1}\right\rangle_{g}$ via Step2 from the result of last case after doing Step1. We have not considered other cases here, because the computation will be more complicated. We hope to implement this algorithm on a computer.

We note that, the recursion formulas for $\left\langle\tau_{b_{L}} \lambda_{g-1}\right\rangle_{g}$ [18] and $\left\langle\tau_{b_{L}} \lambda_{g} \lambda_{1}\right\rangle_{g}$ in Theorem 1.5 have the similar recursion structure. For a given $g$, Hodge integral with $l$ points will reduce to 1 points after only $l$ times recursions. So it is a very 
effective recursion relation. In fact, many Hodge integral recursions have such structure if they are calculated by above algorithm. We formulate the following combinatoric problem.

Let $Q_{l}\left(b_{L}\right)=Q_{l}\left(b_{1}, b_{2}, . ., b_{l}\right)$ be a symmetric function on $\left(b_{1}, b_{2}, . ., b_{l}\right) \in\left(\mathbb{Z}^{+}\right)^{l}$ which is defined by the following recursion relation. For given $g, k$,

$$
Q_{1}(b)= \begin{cases}\text { constant, } & b=3 g-2-k \\ 0, & \text { others. }\end{cases}
$$

$Q_{l}\left(b_{L}\right)= \begin{cases}\sum_{1 \leq i<j \leq l} Q_{l-1}\left(b_{i}+b_{j}-1, b_{L \backslash\{i, j\}}\right) A_{l}\left(b_{L}\right)+B_{l}\left(b_{L}\right) & \sum_{i=1}^{l} b_{i}=3 g-3+l-k, \\ 0, & \text { others. }\end{cases}$

where $A_{l}\left(b_{L}\right)=A_{l}\left(b_{1}, . ., b_{l}\right)$ and $B_{l}\left(b_{L}\right)=B_{l}\left(b_{1}, . ., b_{l}\right)$ are some fixed functions defined on $\left(b_{1}, b_{2}, . ., b_{l}\right) \in\left(\mathbb{Z}^{+}\right)^{l}$.

It is interesting to study the properties of $Q_{l}\left(b_{L}\right)$ defined above. We hope that such combinatoric structure of Hodge integral will be studied further in future.

\section{REFERENCES}

[1] V. Bouchard, A. Klemm, M. Mariño, and S. Pasquetti, Remodeling the B-model, Commun. Math. Phys. 287, 117-178 (2009).

[2] V. Bouchard and M. Mariño, Hurwitz numbers, matrix models and enumerative geometry, Proc. Symposia Pure Math. 78, 263-283 (2008).

[3] L. Chen, Symmetrized cut-join equation of the Mariño-Vafa formula, Pacific Journal of Math. 235, No.2, 201-212 (2008).

[4] L. Chen, Bouchard-Klemm-Mariño-Pasquetti Conjecture for $\mathbb{C}^{3}$, arXiv:0910.3739v1.

[5] L. Chen, Y. Li and K. Liu, Localization, Hurwitz numbers and the Witten conjecture, Asian J. Math. 12 No.4, 511-518 (2008).

[6] T. Ekedahl, S. Lando, M. Shapiro and A. Vainshtein, Hurwitz numbers and intersections on moduli spaces of curves, Invent. Math. 146 No. 2, 297-327 (2001).

[7] B. Eynard, M. Mulase and B. Safnuk, The Laplace transform of the cut-and-join equation and the Bouchard-Mariño conjecture on Hurwitz numbers, math.AG/0907.5224v1

[8] C. Faber and R. Pandharipande, Hodge integrals, partition matrices, and the $\lambda_{g}$ conjecture, Ann. Math.(2) 157 No.1, 97-124 (2003).

[9] I.P. Goulden, D.M. Jackson and R. Vakil, A short prooof of the $\lambda_{g}$-Conjecture without Gromov-Witten theory: Hurwitz theory and the moduli of curves, math.AG/0604297. 
[10] Y. Li, Some results of the Mariño-Vafa Formula, Math. Res. Lett. 13, 10001-10018 (2006).

[11] C.-C. Liu, K. Liu and J. Zhou, A proof of a conjecture of Mariño-Vafa on Hodge integrals, J. Differential Geom. 65 (2003).

[12] C.-C. Liu, K. Liu and J. Zhou, Mariño-Vafa formula and Hodge integral identities, J. Algebric Geom. 15, 379-398 (2006).

[13] M. Mariño and C. Vafa, Framed knots at large N, Orbifolds in mathematics and physics (Madison, WI, 2001), 185-204, Contemp. Math., 310, Amer. Math. Soc., Providence, RI, 2002.

[14] M. Mulase and N. Zhang, Polynomial recursion formula for linear Hodge integrals, Communications in Number Theory and Physics 4 No.2, 267-294 (2010).

[15] H. Xu, Private discussion, 2010.

[16] J. Zhou, Hodge integrals, Hurwitz numbers, and symmetric groups, math.AG/0308024.

[17] J. Zhou, Local Mirror Symmetry for One-Legged Topological Vertex, arXiv:0910.4320.

[18] S. Zhu, Hodge integral with one $\lambda$-class. Sci. China Math. 55 No.1 (2012), 119-130.

\section{Shengmao Zhu}

Department of Mathematics and Center of Mathematical Sciences, Zhejiang University,

Hangzhou, Zhejiang 310027, China

E-mail: zhushengmao@gmail.com 